\title{
The Kelvin-Helmholtz Instability of Momentum Sheets in the Euler Equations for Planar Diffeomorphisms*
}

\author{
Robert I. McLachlan ${ }^{\dagger}$ and Stephen R. Marsland ${ }^{\ddagger}$
}

\begin{abstract}
The Euler equations that describe geodesics on the group of diffeomorphisms of the plane admit singular solutions in which the momentum is concentrated on curves, the so-called momentum sheets analogous to vortex sheets in the Euler fluid equations. We study the stability of straight and circular momentum sheets for a large family of metrics. We prove that straight sheets moving normally to themselves under an $H^{1}$ metric, corresponding to peakons for the one-dimensional (1D) CamassaHolm equation, are linearly stable in Eulerian coordinates, suffering only a weak instability of Lagrangian particle paths, while most other cases are unstable but well-posed. Expanding circular sheets are algebraically unstable for all metrics. The evolution of the instabilities are followed numerically, illustrating several typical dynamical phenomena of momentum sheets.
\end{abstract}

Key words. Euler equations, planar diffemorphisms, momentum sheets, Kelvin-Helmholtz instability

AMS subject classifications. $37 \mathrm{~K} 65,37 \mathrm{M} 15$

DOI. $10.1137 / 060655808$

1. Introduction. The Kelvin-Helmholtz instability of shear layers, which occurs when one fluid layer slides over another of the same or different density, is one of the most famous and ubiquitous instabilities in fluid mechanics. Although studied intensively since its discovery by Kelvin in 1868 and Helmholtz in 1871, it is not yet fully understood. One key idealization is to consider the motion of a vortex sheet, which represents the limit of a thin shear layer in an incompressible, inviscid, two-dimensional (2D) fluid. Early simulations showing the instability developing into a chain of spiral vortices were confounded when Derek Moore showed in 1979 [20] that the sheet in fact develops an infinite curvature singularity at a finite time, a time even before the first vortices appear. Subsequent studies $[10,13,14]$ have focused on regularized versions of the equations of motion and their relationship to weak solutions of the Euler fluid equations.

In this paper we study the analogous, Kelvin-Helmholtz-like instability for a related family of equations, the Euler equations for diffeomorphisms:

$$
\dot{m}+u \cdot \nabla m+\nabla u^{T} \cdot m+m(\operatorname{div} u)=0
$$

where $\dot{m}$ denotes differentiation with respect to time, $u(x, t)\left(u, x \in \mathbb{R}^{n}, t \in \mathbb{R}\right)$ is a velocity

${ }^{*}$ Received by the editors March 31, 2006; accepted for publication (in revised form) by J. Marsden August 28, 2006; published electronically December 5, 2006. This research was supported by the Marsden Fund and the New Zealand Institute of Mathematics and Its Applications. http://www.siam.org/journals/siads/5-4/65580.html

${ }^{\dagger}$ Mathematics Department, Massey University, Palmerston North, New Zealand (r.mclachlan@massey.ac.nz).

${ }^{\ddagger}$ Institute of Information Science, Massey University, Palmerston North, New Zealand (s.r.marsland@massey.ac. $\mathrm{nz})$. 
field, and $m(x, t)$ its associated momentum. The velocity $u$ and momentum $m$ are related by

$$
m=\mathcal{A} u
$$

where $\mathcal{A}$ is an elliptic operator (e.g., $\left.\mathcal{A}=\left(1-\nabla^{2}\right)^{k}\right)$ called the inertia operator.

It was Arnold's celebrated discovery [1] that the Euler fluid equations describe geodesics on the group of volume-preserving (i.e., incompressible) diffeomorphisms. Similarly, (1.1), (1.2) describe geodesics on the group of all diffeomorphisms. There has been much recent interest in the Euler equations (1.1), (1.2) because they arise in several different fields, including computer vision and fluid dynamics. In computer vision they appear in two and three dimensions in the field of image registration, such as in the averaged template matching equations [19, 22] and the geodesic interpolating clamped-plate spline [16], while in fluid dynamics they coincide with the Camassa-Holm wave equation for particular choices of $\mathcal{A}[5]$.

Euler equations such as (1.1) have a natural geometric origin, which we sketch here although these details are not needed in the paper $[2,12,15]$. Let $\mathfrak{G}$ be a Lie or diffeomorphism group with Lie algebra $\mathfrak{g}$. Let $\mathfrak{G}$ be equipped with a left- or right-invariant metric that restricts to a metric $\langle\langle\rangle$,$\rangle on \mathfrak{g}$. Typically, this metric is defined by a linear inertia operator $\mathcal{A}: \mathfrak{g} \rightarrow \mathfrak{g}^{*}$ via

$$
\langle\langle u, v\rangle\rangle:=\langle u, \mathcal{A} v\rangle
$$

The geodesic equation on $T \mathfrak{G}$ can be reduced to give a noncanonical Hamiltonian evolution equation on $\mathfrak{g}$ called the Euler-Poincaré equation, or transferred (via the Legendre transform, $\mathfrak{g} \mapsto \mathfrak{g}^{*}$, which in this case is $u \rightarrow m:=\mathcal{A} u$ ) to a Lie-Poisson system on $\mathfrak{g}^{*}$ called the Euler equations

$$
\dot{m}= \pm \mathrm{ad}_{\mathcal{A}^{-1} m}^{*} m
$$

where the sign is + for left- and - for right-invariant metrics. The variable $m \in \mathfrak{g}^{*}$ is called the momentum and $u \in \mathfrak{g}$ the velocity.

The most famous Euler equation on a Lie group is the equation of motion of a free rigid body $(\mathfrak{G}=S O(3), m=$ body angular momentum). An infinite-dimensional example is the Landau-Lifshitz equation on the loop group $\mathfrak{G}=C^{\infty}(\mathbb{R}, S O(3))$. Examples on diffeomorphism groups include the Euler fluid equations on the group $\mathfrak{G}=\operatorname{Diff}_{\mathrm{vol}}\left(\mathbb{R}^{n}\right)$ of volume-preserving diffeomorphisms with respect to the $L^{2}$ metric ( $m=$ vorticity) [2], the Camassa-Holm equation $\left(\mathfrak{G}=\operatorname{Diff}\left(\mathbb{S}^{1}\right), H^{1}\right.$ metric) $[5]$, and the second-grade fluid equations $\left(\mathfrak{G}=\operatorname{Diff} \mathrm{vol}\left(\mathbb{R}^{n}\right)\right.$, $H^{1}$ metric) [24].

For $\mathfrak{G}=\operatorname{Diff}\left(\mathbb{R}^{n}\right)$ the Euler equations are given by (1.1), (1.2) (see [2, 5, 22] for further details). The inverse of the inertia operator $\mathcal{A}$ is given by convolution with the Green's function $\mathbf{G}$ of $\mathcal{A}$, i.e., $u=\mathbf{G} * m$, where $*$ denotes convolution and $\mathcal{A} \mathbf{G}\left(x, x^{\prime}\right)=\delta\left(x-x^{\prime}\right)$ for $x, x^{\prime} \in \mathbb{R}^{n}$. We shall only consider Euclidean-invariant and diagonal $\mathcal{A}$ (i.e., $\mathcal{A}=a\left(-\nabla^{2}\right) I$ for some function $a$ ); in this case $\mathbf{G}\left(x, x^{\prime}\right)=G\left(\left\|x-x^{\prime}\right\|\right)$ for a scalar function $G$, which we call the kernel. Examples in the literature include Gaussian [7] and Bessel function kernels [24] associated with the $H^{1}$ metric and a family of $H^{k}$ metrics [17]. We will study (1.1), (1.2) for arbitrary Euclidean-invariant operators $\mathcal{A}$. 
A striking feature of Euler equations on diffeomorphism groups is that they admit (formally, at least) exact solutions in which the momentum is concentrated on a submanifold of dimension $l<n$ [5]. The submanifold $M$ is embedded in $\mathbb{R}^{n}$, say, $q: M \times \mathbb{R} \rightarrow \mathbb{R}^{n}$, and the momentum field is given by

$$
m(x, t)=\int_{M} \delta(x-q(s, t)) p(s, t) \mathrm{d} s,
$$

where $p(s, t)$ is the "strength" of the momentum (vorticity in the fluid case) on the submanifold. For the Euler fluid equations these solutions are point vortices for $l=0$, vortex sheets for $l=n-1$, and vortex filaments for $l=1, n=3$. These are widely studied both in their own right and as a means of approximating the evolution of smooth or other vorticities [14].

For the 2D and three-dimensional (3D) Euler fluid equations, convergence of the point vortex solutions to solutions for smooth initial data has been established [14]. The speed of convergence can be improved by smoothing out the point vortices to vortex blobs, even though the (e.g., Gaussian) blobs are no longer exact solutions of the Euler equations. Instead, their evolution can be regarded as that of delta-functions under a slightly different inertia operator. Altering the shape of the blob (which corresponds to the kernel) is equivalent to altering the metric. In this way we are led to consider a wide class of metrics.

Very little is known about the Euler equations (1.1), (1.2). Most studies have taken $\mathcal{A}=\left(1-\alpha^{2} \nabla^{2}\right)$, i.e., an $H^{1}$ metric. In one dimension this gives the Camassa-Holm equation, which is completely integrable. Here the codimension- 1 singular solutions are known to play a special role: they are solitons ( $m$ a sum of delta functions, $u$ a sum of shifted and scaled "peakons" $e^{-|x|}$ ) and are stable in the sense that the long-time solution for initial data that decays to zero at infinity contains only such solitons $[5,8]$. Extensive simulations which have been performed with the same metric in dimensions 2 and 3 [9] suggest that bump-like initial data also evolves toward codimension-1 singular solutions, i.e., momentum sheets. These nearsheets interact with each other and can even undergo reconnection events. The sheets appear to be stable objects, even asymptotically stable, attracting a substantial part of phase space. These numerical results suggest that to understand the behavior of (1.1), (1.2) it is crucial to first understand the behavior of their momentum sheet solutions.

From now on we consider momentum sheets in two dimensions, i.e., $n=2$ and $\operatorname{dim} M=1$.

For such momentum-sheet data the Euler equations form a canonical Hamiltonian system with configuration space the space of embeddings of $M$ in $\mathbb{R}^{2}$, i.e., $Q=\operatorname{Emb}\left(M, \mathbb{R}^{2}\right)$ and phase space $T^{*} Q[5]$. In coordinates, the positions $q(s, t) \in \mathbb{R}^{2}$ and conjugate momenta $p(s, t) \in \mathbb{R}^{2}$, where the parameter $s \in M$ labels points on the sheets. For $n=2, l=1, M$ is a union of open or closed curves. The Hamiltonian is the kinetic energy

$$
H=\frac{1}{2} \iint_{M \times M} p(s)^{T} \mathbf{G}(q(s)-q(t)) p(u) \mathrm{d} s \mathrm{~d} u
$$

and the equations of motion are

$$
\begin{aligned}
& \dot{q}=\int_{M} \mathbf{G}(q(s)-q(u)) p(u) \mathrm{d} u, \\
& \dot{p}=-\int_{M} p(s)^{T} \nabla \mathbf{G}(q(s)-q(u)) p(u) \mathrm{d} u
\end{aligned}
$$


or, for Euclidean-invariant and diagonal G,

$$
\begin{aligned}
H & =\frac{1}{2} \iint_{M \times M} G(r) p(s) \cdot p(u) \mathrm{d} s \mathrm{~d} u, \\
\dot{q} & =\int_{M} G(r) p(u) \mathrm{d} u, \\
\dot{p} & =-\int_{M} p(s) \cdot p(u) G^{\prime}(r) \frac{\mathbf{r}}{r} \mathrm{~d} u,
\end{aligned}
$$

where

$$
\mathbf{r}=q(s)-q(u), \quad r=\|\mathbf{r}\|
$$

If the metric is Euclidean-invariant then (1.1), (1.2), and (1.8) are too, and one can, via a symmetry reduction, study the behavior of translation or rotation-invariant initial data. This is carried out for sets of parallel straight and concentric circular momentum sheets in [6]. Our goal here is to investigate the stability of these solutions in the simplest settings of either (i) a single straight sheet, moving at a certain angle to itself, or (ii) a single expanding circular sheet.

The motion of straight vortex sheets for the Euler fluid equations, subject to the classical Kelvin-Helmholtz instability, is known to be ill-posed [14]. The growth rate of Fourier mode $\omega$ is $\mathcal{O}(\omega)$, so that even for analytic initial data, eventually analyticity is lost and the (classical) solution terminates in a singularity. The growth rates can be moderated by incorporating either a physical regularizing effect, such as surface tension [10] or altering the metric from $L^{2}$ to $H^{1}$ to obtain the Euler- $\alpha$ or second-grade fluid equations [11], or a nonphysical regularization, such as smoothing the kernel.

We shall see that straight momentum sheets are also unstable for the diffeomorphism case, and because of the close analogy in the geometric structure of the equations and of the initial data, we shall call it a Kelvin-Helmholtz instability. However, there are several key differences between the fluid and diffeomorphism cases. The latter has no volume-preserving constraint and more degrees of freedom (the sheet strength is constant for fluids, while $p(x, t) \in \mathbb{R}^{2}$ for diffeomorphisms); these make the diffeomorphism case more unstable. But it has more filtering of the high modes in its inertia operator $(\mathcal{A}=1$ for the fluid case, while typically $\mathcal{A}=\left(1-\nabla^{2}\right)^{k}$ for diffeomorphisms), which makes the diffeomorphism case more stable. The stability and well-posedness of the diffeomorphism case is therefore difficult to predict.

Stretched circular vortex sheets, on the other hand, are stable if the stretching is sufficiently strong. (A single vortex sheet does not expand by itself but can be made to expand by placing a line source at the origin.) They are well-posed but unstable under slow expansion, and stable under fast expansion [21]. We expect to see similar behavior here, especially because the numerical solutions for smooth initial data tend to show curved sheets expanding radially.

In section 2 we describe the particle-relabeling symmetry of (1.7) and its associated conserved quantity, the potential vorticity $q_{s} \cdot p$. In section 3.1 we linearize the equations of motion (1.8), giving a system of four linear integro-differential equations. Remarkably, for straight sheets they can be solved, giving a complete and explicit determination of linear stability for all metrics (section 3.2). One case, of a straight sheet moving normally to itself under an $H^{1}$ 
metric (section 3.3), is only algebraically unstable, and we prove in Proposition 3.9 that even this instability is confined to the Lagrangian particle motion; the Eulerian motion of the sheet is linearly stable. In section 3.4 we follow the evolution of the unstable cases numerically. For circular sheets (section 4) the linearized equations are even worse, having nonconstant coefficients in space and time. The time variation can only be dealt with by taking the limit of large radius; in this case the dependence on the metric drops out and the resulting PDE can be solved explicitly. We find that radially expanding circular sheets are algebraically unstable for all metrics.

2. Symmetries and conserved quantities. The equations of motion for momentum sheets, (1.7), have a family of conserved quantities arising from a particle-relabeling symmetry. More precisely, the symmetry group $\mathfrak{G}=\operatorname{Diff}(M)$ acts on sheet positions in $Q=\operatorname{Emb}\left(M, \mathbb{R}^{2}\right)$ on the right by composition,

$$
\mathfrak{G} \times Q \rightarrow Q:(\varphi, q(s)) \mapsto q(\varphi(s)),
$$

and on $T^{*} Q$ by cotangent lifts,

$$
\mathfrak{G} \times T^{*} Q \rightarrow T^{*} Q:(\varphi,(q(s), p(s))) \mapsto\left(q(\varphi(s)), p(\varphi(s)) \varphi^{\prime}(s)\right) .
$$

Equation (2.2) is a symplectic map, as can be seen by the fact that it is generated by the generating function $S(q, P)$ as $(q(s), p(s)) \mapsto(Q(s), P(s))$ with

$$
S(q, P)=\int_{M} q(\varphi(s)) P(s) \mathrm{d} s=\int_{M} q(s) P\left(\varphi^{-1}(s)\right) \frac{\mathrm{d} s}{\varphi^{\prime}\left(\varphi^{-1}(s)\right)}
$$

according to

$$
\begin{aligned}
& Q(s)=\frac{\delta S}{\delta P}=q(\varphi(s)) \\
& p(s)=\frac{\delta S}{\delta q}=P\left(\varphi^{-1}(s)\right) \frac{1}{\varphi^{\prime}\left(\varphi^{-1}(s)\right)} \Rightarrow P(s)=p(\varphi(s)) \varphi^{\prime}(s) .
\end{aligned}
$$

The generators of the group action are the vector fields

$$
\begin{aligned}
& \dot{q}=f(s) q_{s}, \\
& \dot{p}=(f(s) p)_{s},
\end{aligned}
$$

where $f(s)=\dot{\varphi}(s)$. These are Hamiltonian vector fields with Hamiltonian $J_{f}=\int_{M} f(s) q_{s}(s)$. $p(s) \mathrm{d} s$, which is therefore the momentum map for the group action. The Hamiltonian (1.6) is invariant under the group action

$$
\begin{aligned}
g \cdot H & =\frac{1}{2} \iint_{M \times M} \varphi^{\prime}(s) p(\varphi(s))^{T} \mathbf{G}(q(\varphi(s))-q(\varphi(u))) p(\varphi(u)) \varphi^{\prime}(u) \mathrm{d} s \mathrm{~d} u \\
& =\frac{1}{2} \iint_{M \times M} p(\tilde{s})^{T} \mathbf{G}(q(\tilde{s})-q(\tilde{u})) p(\tilde{u}) \mathrm{d} \tilde{s} \mathrm{~d} \tilde{u} \\
& =H
\end{aligned}
$$


where we have made the change of variables $\tilde{s}=\varphi(s), \tilde{u}=\varphi(u)$. Therefore, the momentum map $J_{f}$ is a conserved quantity of Hamilton's equations for $H$ for any function $f(s)$. Choosing $f(s)$ to be a delta-function, we get the pointwise conserved quantities $J(s)=q_{s}(s) \cdot p(s)$ for each $s$, whose conservation can be directly checked from the equations of motion. By analogy with the shallow water equations we call $J(s)$ the potential vorticity of the momentum sheet.

It can be seen from $(2.2)$ that the momentum sheet strength $p(s)$ is not a fundamental physical property of the sheet, because it changes when different coordinates are used on the sheet. The fundamental variables of the sheets are the invariants of the group action, namely, the unit tangent vector $q_{s}(s) /\left\|q_{s}(s)\right\|$ and the true momentum $p(s) /\left\|q_{s}(s)\right\|$. The true momentum contains only one independent component because of the conservation of potential vorticity $q_{s}(s) \cdot p(s)$.

If the metric is Euclidean-invariant the equations have four other conserved quantities: the energy $H$, the total momentum $\int p(s) \mathrm{d} s$ (from translation symmetry), and the angular momentum $\int q(s) \times p(s) \mathrm{d} s$ (from rotational symmetry).

\section{Stability of straight sheets.}

3.1. Analysis of the general case. We first determine the linearized equations of motion for straight sheets, which, because of translation symmetry, can be diagonalized by a Fourier transform. Finding the growth rates requires finding the eigenvalues of $4 \times 4$ matrices. However, this is not required in the determination of stability, for which an explicit condition can be found. We give the condition in terms of the critical angle of propagation of the sheet for a disturbance of a given wavenumber to be unstable as a function of the Fourier transform of the Green's function of the metric.

Proposition 3.1. Consider a straight sheet located at $q(s, t)=t U+(0, s)^{\mathrm{T}}$ with constant momentum $p(s, t)=\rho=\left(\rho_{1}, \rho_{2}\right)$ and velocity $U=\left(U_{1}, U_{2}\right)$, where $s$ is the Lagrangian parameter along the sheet. Under a small perturbation to $(q, p)+\varepsilon z(s, t)$, the growth of the perturbation is controlled to leading order by the $4 \times 4$ linear systems

$$
\dot{\tilde{z}}=\left(\begin{array}{cccc}
0 & a \widetilde{G} & \widetilde{G} & 0 \\
0 & b \widetilde{G} & 0 & \widetilde{G} \\
c & 0 & 0 & 0 \\
0 & \left(a^{2}+b^{2}\right) \widetilde{G} & a \widetilde{G} & b \widetilde{G}
\end{array}\right) \tilde{z}
$$

where

$$
\begin{aligned}
\tilde{z}(\omega, t) & =\int_{-\infty}^{\infty} z(s, t) e^{-2 \pi i \omega s} \mathrm{~d} s, \\
\widetilde{G}(\omega) & =\int_{-\infty}^{\infty} G(\omega) e^{-2 \pi i \omega s} \mathrm{~d} s, \\
c & =\left.\rho^{2} \widetilde{F}\right|_{0} ^{\omega}, \\
a & =-2 \pi i \omega \rho_{1}, \\
b & =-2 \pi i \omega \rho_{2}, \\
\rho^{2} & =\rho_{1}^{2}+\rho_{2}^{2}, \quad \text { and }
\end{aligned}
$$




$$
\left.\widetilde{F}\right|_{0} ^{\omega}=4 \pi^{2} \int_{0}^{\omega} \omega^{\prime} \widetilde{G}\left(\omega^{\prime}\right) \mathrm{d} \omega^{\prime} .
$$

The system (3.1) has an eigenvalue with positive real part, indicating an exponential instability in wavenumber $\omega$, if and only if

$$
\Delta:=-1+\left(11 \cos ^{2} \phi-8\right) \mu+\left(\cos ^{4} \phi+12 \cos ^{2} \phi-16\right) \mu^{2}+\left(\cos ^{4} \phi\right) \mu^{3}<0,
$$

where

$$
\mu=\mu(\omega)=\frac{4 \pi^{2} \omega^{2} \widetilde{G}(\omega)}{\left.\widetilde{F}\right|_{0} ^{\omega}}
$$

and

$$
\rho_{1}=\rho \cos \phi, \quad \rho_{2}=\rho \sin \phi
$$

or equivalently, if and only if

$$
\cos ^{2} \phi<\frac{(5+4 \mu)^{3 / 2}-11-12 \mu}{2 \mu(1+\mu)} .
$$

Proof. First note that, for compatibility, $U=G_{0} \rho$, where $G_{0}=\int_{-\infty}^{\infty} G(r) \mathrm{d} r$. We make a small perturbation and let

$$
\begin{aligned}
& q(s, t)=t U+\left(\begin{array}{l}
0 \\
s
\end{array}\right)+\varepsilon u, \\
& p(s, t)=\rho+\varepsilon v .
\end{aligned}
$$

The distance $d$ between two points with parameters $s$ and $\sigma$ on the curve is given by

$$
\begin{aligned}
\|q(s)-q(\sigma)\|_{2}= & {\left[\left(\left(U_{1} t+\varepsilon u_{1}(s)\right)-\left(U_{1} t+\varepsilon u_{1}(\sigma)\right)\right)^{2}\right.} \\
& \left.+\left(\left(U_{2} t+s+\varepsilon u_{2}(s)\right)-\left(U_{2} t+\sigma+\varepsilon u_{2}(\sigma)\right)\right)^{2}\right]^{1 / 2} \\
= & s-\sigma+\varepsilon\left(u_{2}(s)-u_{2}(\sigma)\right)+\mathcal{O}\left(\varepsilon^{2}\right),
\end{aligned}
$$

where $s-\sigma$ is held fixed. The first equation of motion

$$
\dot{q}=\int_{-\infty}^{\infty} G\left(\|q(s)-q(t)\|_{2}\right) p(\sigma) \mathrm{d} \sigma
$$

linearizes to

$$
\begin{aligned}
\dot{u} & =\left.\frac{d}{d \varepsilon}\right|_{\varepsilon=0} \int_{-\infty}^{\infty} G\left(s-\sigma+\varepsilon\left(u_{2}(s)-u_{2}(\sigma)\right)\right)(\rho+\varepsilon v(\sigma)) \mathrm{d} \sigma \\
& =\int_{-\infty}^{\infty}\left(G(s-\sigma) v(\sigma)+\rho G^{\prime}(s-\sigma)\left(u_{2}(s)-u_{2}(\sigma)\right)\right) \mathrm{d} \sigma \\
& =\int_{-\infty}^{\infty}\left(G(s-\sigma) v(\sigma)-\rho G^{\prime}(s-\sigma) u_{2}(\sigma)\right) \mathrm{d} \sigma,
\end{aligned}
$$


where in the last line we have used that $\int_{-\infty}^{\infty} G^{\prime}(\sigma) \mathrm{d} \sigma=0$ because $G(\sigma)$ is an even function. As the right-hand side is a convolution, it is diagonalized by the Fourier transform, giving

$$
\dot{\tilde{u}}=\widetilde{G}\left(\tilde{v}-2 \pi i \omega \tilde{u}_{2} \rho\right) .
$$

(We are using the Fourier transform pair $\tilde{u}(\omega)=\int_{-\infty}^{\infty} u(x) e^{-2 \pi i \omega x} \mathrm{~d} x, u(x)=\int_{-\infty}^{\infty} \tilde{u}(\omega) e^{2 \pi i \omega x} \mathrm{~d} \omega$.) Introducing $F(r)=G^{\prime}(r) / r$, the momentum equation is

$$
\begin{aligned}
\dot{p} & =-\int_{-\infty}^{\infty}(p(s) \cdot p(\sigma)) \nabla G\left(\|q(s)-q(\sigma)\|_{2}\right) \mathrm{d} \sigma \\
& =-\int_{-\infty}^{\infty}(p(s) \cdot p(\sigma)) F\left(\|q(s)-q(\sigma)\|_{2}\right)(q(s)-q(\sigma)) \mathrm{d} \sigma,
\end{aligned}
$$

which linearizes to

$$
\begin{aligned}
\dot{v}=- & \left.\frac{d}{d \varepsilon}\right|_{\varepsilon=0} \int_{-\infty}^{\infty}\left[\left(\rho_{1}+\varepsilon v_{1}(s)\right)\left(\rho_{1}+\varepsilon v_{1}(\sigma)\right)+\left(\rho_{2}+\varepsilon v_{2}(s)\right)\left(\rho_{2}+\varepsilon v_{2}(\sigma)\right)\right] \\
& F\left(s-\sigma+\varepsilon\left(u_{2}(s)-u_{2}(\sigma)\right)\right)\left(\begin{array}{c}
\varepsilon\left(u_{1}(s)-u_{1}(\sigma)\right) \\
s-\sigma+\varepsilon\left(u_{2}(s)-u_{2}(\sigma)\right)
\end{array}\right) \mathrm{d} \sigma \\
=- & \int_{-\infty}^{\infty}\left(\begin{array}{c}
\rho^{2} F(s-\sigma)\left(u_{1}(s)-u_{1}(\sigma)\right) \\
(\rho \cdot v(\sigma)) G^{\prime}(s-\sigma)+\rho^{2} G^{\prime \prime}(s-\sigma)\left(u_{2}(s)-u_{2}(\sigma)\right)
\end{array}\right) \mathrm{d} \sigma
\end{aligned}
$$

which is diagonalized by the Fourier transform to become

$$
\begin{aligned}
& \dot{\tilde{v}}_{1}=\left.\rho^{2} \widetilde{F}\right|_{0} ^{\omega} \tilde{u}_{1}, \\
& \dot{\tilde{v}}_{2}=-\left(2 \pi i \omega(\rho \cdot \tilde{v})+(2 \pi \omega)^{2} \rho^{2} \tilde{u}_{2}\right) \widetilde{G} .
\end{aligned}
$$

Here we have used, for example, $\int_{-\infty}^{\infty} F(\sigma) \mathrm{d} \sigma=\widetilde{F}(0)$. The diagonalized, linearized equations of motion (3.9), (3.12) are given collectively by the $4 \times 4$ linear systems in (3.1). The expression for $\left.\widetilde{F}\right|_{0} ^{\omega}$ in terms of $\widetilde{G}(\omega)$ is easily established by manipulating the Fourier transforms.

The characteristic polynomial of the coefficient matrix of (3.1) is

$$
\begin{aligned}
P(\lambda) & =\lambda\left(\left(c \widetilde{G}-\lambda^{2}\right)(2 b G-\lambda)-a^{2} \widetilde{G}^{2} \lambda\right) \\
& =\lambda\left(\left(\left.\rho^{2} \widetilde{F}\right|_{0} ^{\omega} \widetilde{G}-\lambda^{2}\right)\left(-4 \pi i \omega \rho_{2} \widetilde{G}-\lambda\right)+4 \pi^{2} \omega^{2} \rho_{1}^{2} \widetilde{G}^{2} \lambda\right) .
\end{aligned}
$$

Although $P(\lambda)$ has complex coefficients, $i P(i \lambda)$ has real coefficients. If the cubic $i P(i \lambda) / \lambda$ has one real and two complex conjugate roots, then $P(\lambda)$ has precisely one root with a positive real part, indicating instability. This is true if and only if the discriminant of the cubic is negative. This discriminant, after removing the positive factor (see Proposition 3.5 and Corollary 3.6 below) $4\left(\left.\widetilde{G} \widetilde{F}\right|_{0} ^{\omega}\right)^{3}$ and expressing it in terms of the new variable $\mu(\omega)$, is $\Delta$ in (3.2).

The stability criterion (3.4) is then determined simply by solving a quadratic equation.

Note that (3.1) is a complex Hamiltonian system, with conjugate variables $\tilde{u}(\omega)$ and $\overline{\tilde{v}}(\omega)$, so its eigenvalues do not obey the usual $(\lambda,-\lambda, \bar{\lambda},-\bar{\lambda})$ symmetry. When written as an $8 \times 8$ real Hamiltonian system, all the eigenvalues given below are paired with their complex conjugates. 


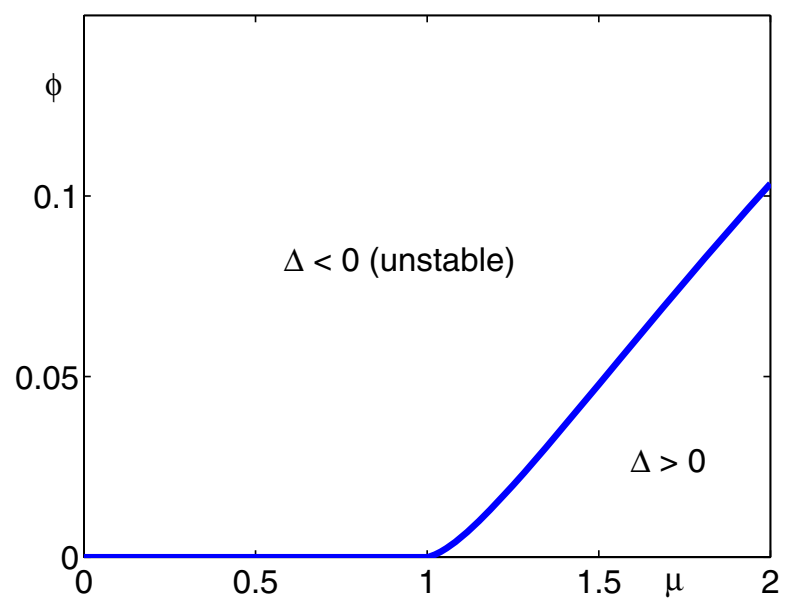

Figure 1. The stability region for straight sheets in terms of $\phi$, the angle of propagation of the sheet to its normal (i.e., $\rho_{1}=\rho \cos \phi, \rho_{2}=\rho \sin \phi$ ), and $\mu(\omega)=\omega^{2} \widetilde{G}(\omega) / \int_{0}^{\omega} w^{\prime} \widetilde{G}\left(\omega^{\prime}\right) \mathrm{d} \omega^{\prime}$. Here $\omega$ is the wavenumber of the disturbance and $\widetilde{G}$ is the Fourier transform of the Green's function of the metric. Since typically $\mu \leq 2$, only sheets moving nearly normal to themselves can be stable at any wavenumber.

Note from (3.13) that $\lambda=0$ is always an eigenvalue. This corresponds to the particlerelabeling symmetry and its associated conserved quantity, the potential vorticity $J(s)$.

The instability region in terms of $\phi$ and $\mu$ is shown in Figure 1. For the metrics we will consider, it is always the case that $0<\mu(\omega) \leq 2$ for all $\omega$.

Corollary 3.2. $\Delta=-1$ at $\mu=0$ for all $\phi$, indicating instability when $\mu$ is sufficiently small.

(We shall see below that for all $H^{k}$ metrics with $k>\frac{3}{2}, \mu(\infty)=0$; i.e., all such straight sheets are unstable at high wavenumbers.)

Corollary 3.3. Stability can be lost, but never gained, with increasing angle of propagation $\phi$.

Proof. The coefficients of $\mu$ in $\Delta(3.2)$ are all nonincreasing functions of $\phi \in[0, \pi / 2]$.

Corollary 3.4. The eigenvalues can be reduced to the solution of a quadratic in two cases, when $\rho_{1}=0$ and when $\rho_{2}=0$.

When $\rho_{2}=0$ the sheet is moving perpendicularly to itself and the eigenvalues are $(0,0, \lambda,-\lambda)$, where

$$
\lambda^{2}=\widetilde{G}\left(c+a^{2} \widetilde{G}\right)=\widetilde{G}\left(\left.\widetilde{F}\right|_{0} ^{\omega}-(2 \pi \omega)^{2} \widetilde{G}\right)=\left.\widetilde{G} \widetilde{F}\right|_{0} ^{\omega}(1-\mu) .
$$

The double zero eigenvalue has a single eigenvector and hence generates an algebraic $\mathcal{O}(t)$ instability for all $G$. Exponential stability, on the other hand, is determined by $\mu$ and depends on a subtle balance between the Fourier transforms of $G(r)$ and $G^{\prime}(r) / r$.

When $\rho_{1}=0$ the sheet is moving tangentially to itself and the eigenvalues are

$$
\left(0,-4 \pi i \rho_{2} \omega \widetilde{G}, \pm \rho_{2} \sqrt{\left.\widetilde{F}\right|_{0} ^{\omega} \widetilde{G}}\right) .
$$

We will consider Euclidean-invariant metrics of the form $\mathcal{A}=a\left(-\nabla^{2}\right)$, where $a\left(\eta^{2}\right)$ is the Fourier symbol of the operator. For example, for the $H^{1}$ metric $1-\nabla^{2}$, we have $a(\eta)=1+\eta$. For such metrics we can easily calculate both the Green's function and its Fourier transform. 
Proposition 3.5. The Green's function $G(\|x\|)$ of the operator $\mathcal{A}=a\left(-\nabla^{2}\right)$ and its Fourier transform $\widetilde{G}(\omega)$ are given by

$$
G(r)=\frac{1}{2 \pi} \int_{0}^{\infty} \frac{1}{a\left(\eta^{2}\right)} J_{0}(\eta r) \eta \mathrm{d} \eta
$$

(where $J_{0}$ is the zero order Bessel function of the first kind) and

$$
\widetilde{G}(\omega)=\frac{1}{\pi} \int_{0}^{\infty} \frac{\mathrm{d} \nu}{a\left(\nu^{2}+(2 \pi \omega)^{2}\right)},
$$

respectively.

Proof. A Hankel transform of the radially symmetric PDE $\mathcal{A} G=\delta(\|x\|)$ gives (3.15) (see, e.g., [25]). We then have

$$
\begin{aligned}
& \widetilde{G}(\omega)=\int_{-\infty}^{\infty} G(r) e^{-2 \pi i \omega r} \mathrm{~d} r \\
& =2 \int_{0}^{\infty} G(r) \cos (2 \pi \omega r) \mathrm{d} r \\
& =\frac{1}{\pi} \int_{0}^{\infty} \mathrm{d} r \int_{0}^{\infty} \mathrm{d} \eta J_{0}(\eta r) \eta \cos (2 \pi \omega r) \frac{1}{a\left(\eta^{2}\right)} \\
& =\frac{1}{\pi} \int_{2 \pi \omega}^{\infty} \frac{\eta \mathrm{d} \eta}{a\left(\eta^{2}\right) \sqrt{\eta^{2}-(2 \pi \omega)^{2}}} \\
& =\frac{1}{\pi} \int_{0}^{\infty} \frac{\mathrm{d} \nu}{a\left(\nu^{2}+(2 \pi \omega)^{2}\right)}
\end{aligned}
$$

because

$$
\int_{0}^{\infty} J_{0}(\eta r) \cos (2 \pi \omega r) \mathrm{d} r= \begin{cases}\frac{1}{\sqrt{\eta^{2}-(2 \pi \omega)^{2}},} & 0<2 \pi \omega<\eta \\ 0, & \eta<2 \pi \omega .\end{cases}
$$

Here $\nu^{2}=\eta^{2}-(2 \pi \omega)^{2}$.

Apart from being an easy way to calculate $\widetilde{G},(3.16)$ has the following immediate consequences.

Corollary 3.6. For any Euclidean-invariant, diagonal metric, straight sheets moving tangentially to themselves are linearly unstable at all positive wavenumbers.

Proof. From positive-definiteness of the metric, $a(\nu)>0$ for all $\nu$ and hence $\widetilde{G}(\omega)>0$. Therefore $\left.\widetilde{F}\right|_{0} ^{\omega}>0$ for all $\omega>0$ and the mode with eigenvalue $\left|\rho_{2}\right| \sqrt{\left.\widetilde{F}\right|_{0} ^{\omega} \widetilde{G}}$ is unstable.

Corollary 3.7. For any $H^{k}$ metric, $a(\nu)=\mathcal{O}\left(\nu^{k}\right)$ as $n \rightarrow \infty$ and hence $\widetilde{G}$ exists and is $\mathcal{O}\left(\omega^{1-2 k}\right)$ as $\omega \rightarrow \infty$ if $k>\frac{1}{2}$. Similarly, $\left.\widetilde{F}\right|_{0} ^{\omega}$ exists and is $\mathcal{O}\left(\omega^{3-2 k}\right)$ for $\frac{1}{2}<k<\frac{3}{2}$ and $\mathcal{O}(1)$ for $k>\frac{3}{2}$. Consequently, the unstable eigenvalue for tangentially moving sheets is $\mathcal{O}\left(\omega^{2-2 k}\right)$ for $\frac{1}{2}<k<\frac{3}{2}$ and $\mathcal{O}\left(\omega^{(1-2 k) / 2}\right)$ for $k>\frac{3}{2}$. Therefore (i) for $\frac{1}{2}<k<1$ the equation is linearly ill-posed for initial data in any fixed Sobolev space; (ii) for $k \geq 1$ the growth rates are bounded for large $\omega$ and hence do not lead to linear ill-posedness. 

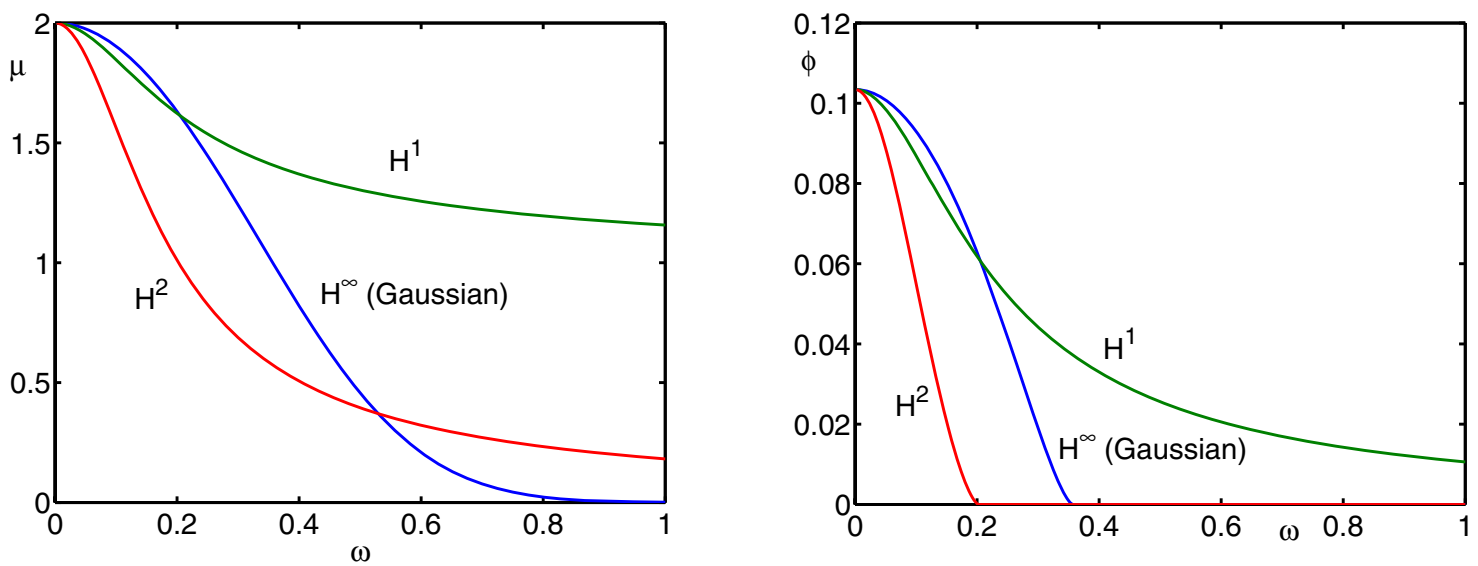

Figure 2. Left: Each kernel $G(r)$ determines a function $\mu(\omega)$ which determines the stability of a disturbance with wavenumber $\omega$ (see (3.3), (3.4), and Figure 1). Right: The resulting stability regions for each metric. Here $\phi(\omega)$ is the critical angle of propagation of a straight sheet to its normal above which a disturbance with wavenumber $\omega$ is unstable.

Note that the critical case $k=1$ is interesting, however, because then the eigenvalue tends to a constant as $\omega \rightarrow \infty$. For $k>1$ the instability will manifest itself at the frequency with the maximum eigenvalue and then follow an essentially unique nonlinear evolution, forgetting the initial perturbation, while for $k=1$ all high frequencies grow at the same rate and the nonlinear evolution depends on the initial perturbation.

Corollary 3.8. For any $H^{k}$ metric, as $\omega \rightarrow \infty$ we have $\mu=\mathcal{O}(1)$ for $\frac{1}{2}<k<\frac{3}{2}$ and $\mu=\mathcal{O}\left(\omega^{3-2 k}\right)$ for $k>\frac{3}{2}$. Therefore, for sheets moving normally to themselves, all sufficiently large wavenumbers are unstable for all $H^{k}$ metrics with $k>\frac{3}{2}$.

\subsection{Stability under various metrics.}

Case 1. Gaussian metric. We consider the $H^{\infty}$ metric $\mathcal{A}=\exp \left(-\nabla^{2} / 4\right)$, with Fourier symbol $a\left(\nu^{2}\right)=\exp \left(\nu^{2} / 4\right)$, for which an application of (3.15) and (3.16) gives the Gaussian kernel

$$
G(r)=\frac{1}{\pi} e^{-r^{2}}
$$

and

$$
\begin{aligned}
\widetilde{G} & =\frac{1}{\sqrt{\pi}} e^{-\pi^{2} \omega^{2}}, \\
\left.\widetilde{F}\right|_{0} ^{\omega} & =\frac{2}{\sqrt{\pi}}\left(1-e^{-\pi^{2} \omega^{2}}\right)
\end{aligned}
$$

(see Figure 2). The growth rates for a range of propagation angles $\phi$ are shown in Figure 3. When $\phi=0$, wavenumbers with $0<\omega<0.3568$ (i.e., wavelength more than $1 / 0.3568=$ 2.8027 ) have zero real part and are neutrally stable, while wavenumbers with $\omega>0.3568$ are unstable. The exponential smoothing of the high frequencies by the Green's function is not enough to stabilize these perturbations. Although arbitrarily high frequencies are unstable, 

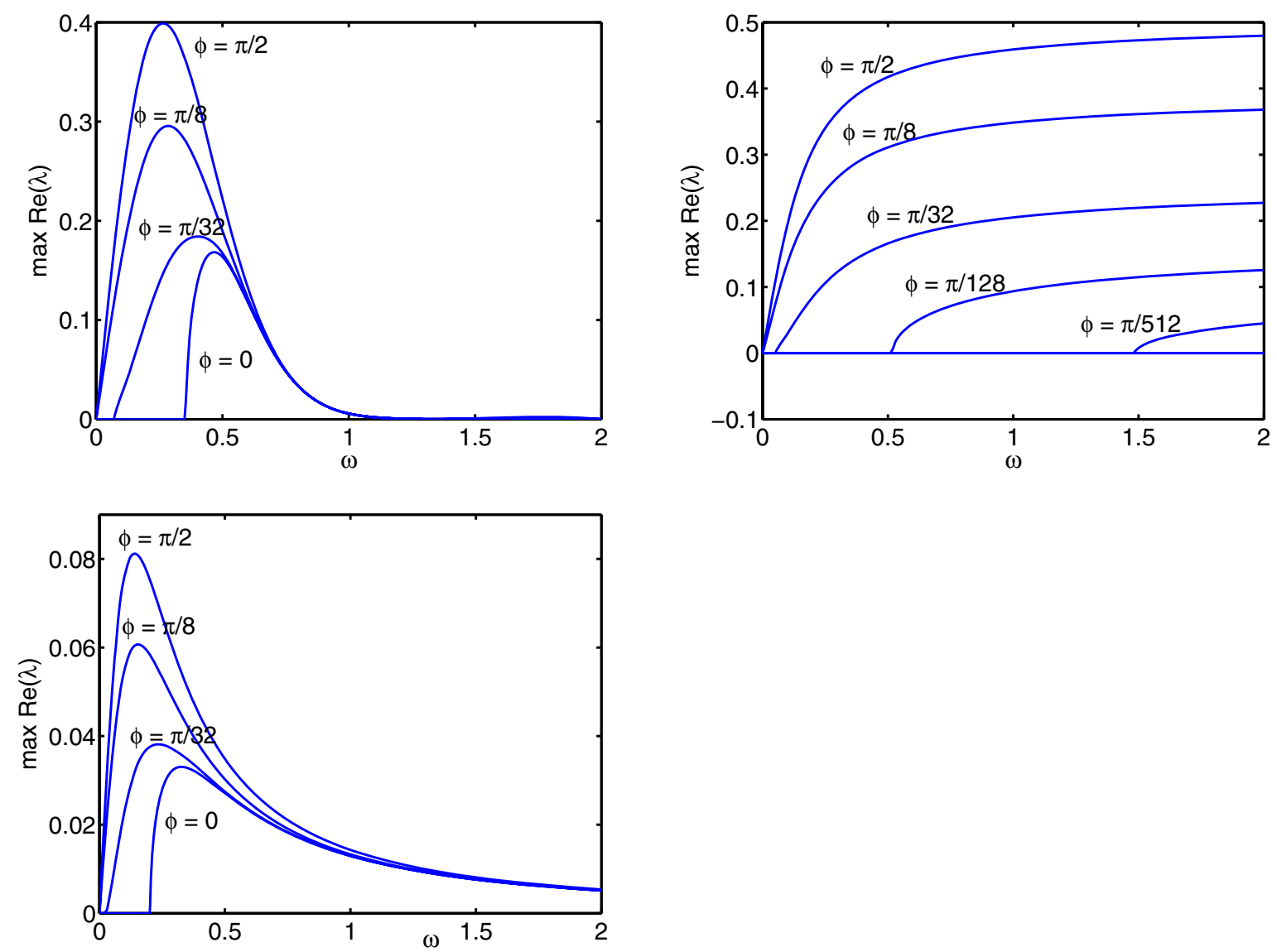

Figure 3. The growth rate of disturbances with wavenumber $\omega$ to a straight sheet with angle of propagation $\phi$ to its normal. Top left: $H^{\infty}$ (Gaussian) metric. Top right: $H^{1}$ metric. Bottom: $H^{2}$ metric.

the exponential smoothing of the high frequencies is reflected in the stability analysis by their exponentially small growth rate. The growth rate is strongly peaked with a maximum of $\lambda=0.1684$ at $\omega=0.4671$.

When $\phi=\pi / 2$, all wavenumbers are unstable, with the growth rate strongly peaked with a maximum of $\lambda=0.3989$ at $\omega=0.2650$.

Case 2. $H^{1}$ metric. For the $H^{1}$ metric $\mathcal{A}=1-\nabla^{2}, a(\nu)=1+\nu$ and an application of (3.15) and (3.16) give (where $K_{0}$ is the zero order modified Bessel function of the second kind)

$$
\begin{aligned}
G(r) & =K_{0}(r) /(2 \pi), \\
\widetilde{G} & =\frac{1}{2 \sqrt{1+(2 \pi \omega)^{2}}}, \\
\left.\widetilde{F}\right|_{0} ^{\omega} & =\frac{1}{2}\left(\sqrt{1+(2 \pi \omega)^{2}}-1\right) .
\end{aligned}
$$


In the eigenvalue calculations, the dominant terms in $\widetilde{G}$ and $\left.\widetilde{F}\right|_{0} ^{\omega}$ cancel out with the following results. When $\phi=0$, the real part of all eigenvalues is zero for all $\omega$. There is no exponential instability - only an algebraic instability due to the double zero eigenvalue. We examine this case in detail in section 3.3, proving a theorem on linear stability. Curiously, although the $H^{1}$ metric applies less smoothing to the momentum than the $H^{\infty}$ metric does, it is in a sense more stable. For $\phi>0$ there is a band of instability in $\left(\omega^{*}, \infty\right)$, where $\omega^{*}$ decreases from $\infty$ to 0 as $\phi$ increases from 0 to 0.1034 . The growth rates tend to a positive constant as $\omega \rightarrow \infty$, indicating that the nonlinear evolution of the instability depends on the high-frequency part of the initial perturbation. For $\phi>0.1034$ all wavenumbers are unstable. The maximum growth rate of $\lambda \rightarrow \frac{1}{2}$ occurs for $\phi=\pi / 2, \omega \rightarrow \infty$.

Case 3. $H^{2}$ metric. For $\mathcal{A}=\left(1-\nabla^{2}\right)^{2}$ an application of (3.15) and (3.16) gives (where $K_{1}$ is the first order modified Bessel function of the second kind)

$$
\begin{aligned}
G(r) & =\frac{1}{4 \pi} r K_{1}(r), \\
\widetilde{G} & =\frac{1}{4\left(4 \pi^{2} \omega^{2}+1\right)^{3 / 2}}, \text { and } \\
\left.\widetilde{F}\right|_{0} ^{\omega} & =\frac{1}{4}\left(1-\frac{1}{\sqrt{4 \pi^{2} \omega^{2}+1}}\right) .
\end{aligned}
$$

The extra smoothing of high wavenumbers in the $H^{2}$ compared to the $H^{1}$ metric is already enough to make the behavior of straight sheets qualitatively the same as the $H^{\infty}$ (Gaussian) case, as the general theory of Corollaries 3.7 and 3.8 indicates. They are exponentially unstable for all $\phi$. For $\phi=0$ all wavenumbers $\omega>0.2024$ are unstable, with the growth rate decaying slowly, $\mathcal{O}\left(\omega^{-3 / 2}\right)$, as $\omega \rightarrow \infty$. The maximum growth rate of only $\operatorname{Re}(\lambda)=0.03305$ comes at $\omega=0.3266$. For $\phi=\pi / 2$, the maximum growth rate is $\lambda=0.08119$ at $\omega=0.1404$.

3.3. Stability of straight sheets in an $\boldsymbol{H}^{1}$ metric. The case $\phi=0$ of a straight sheet moving normally to itself corresponds to the motion of a single peakon in the 1D CamassaHolm equation. This case was shown in section 3.2, Case 2, to have no eigenvalues with positive real parts. The perturbation grows as $\mathcal{O}(t)$ due to the double zero eigenvalue. However, we now show that the algebraic instability applies only to the Lagrangian motion; the Eulerian motion, in which one identifies two sheets related by the particle-relabeling symmetry (2.2), is in fact linearly stable. The only effect of the instability is to push particles along the sheet. To study this, in the following proposition we introduce a norm that measures the perturbation to the true momentum and to the $x$-component of the tangent to the sheet. (Only the $x$ component is relevant to the reduced motion, because $\left.\frac{\mathrm{d}}{\mathrm{d} \varepsilon}\right|_{\varepsilon=0} q_{s} /\left\|q_{s}\right\|=\left(u_{1 s}, 0\right)^{T}$.)

Some care is required because although the Fourier modes are bounded in time, they are not uniformly bounded with respect to $\omega$. It would be possible for the solution itself to grow in time. These considerations dictate the choice of norm in the following proposition. We use the Sobolev norms defined by $\|u\|_{H^{n}}^{2}=\int\left(1+|\omega|^{2 n}\right)|\tilde{u}|^{2} \mathrm{~d} \omega$.

Proposition 3.9. Let $\mathcal{A}=\left(1-\nabla^{2}\right)$ and consider the motion of a straight sheet moving normally to itself: let $q(s, 0)=(0, s)^{T}+\varepsilon u(s, 0), p(s, 0)=(1,0)^{T}+\varepsilon v(s, 0), z=(u, v)^{T}$. Let $j(s)=\left.\frac{\mathrm{d}}{\mathrm{d} \varepsilon}\right|_{\varepsilon=0} J(s)$ be the perturbation to the potential vorticity $J(s)=q_{s} \cdot p$ and $k(s, t)=$ $\left.\frac{\mathrm{d}}{\mathrm{d} \varepsilon}\right|_{\varepsilon=0} p(s, t) /\left\|q_{s}(s, t)\right\|$ be the perturbation to the true momentum. Then for any $n \geq 1$ the 
motion is linearly stable in the norm

$$
\|z\|_{n}=\left\|u_{1 s}\right\|_{H^{n}}+\|k\|_{H^{n+1 / 2}}+\|j\|_{H^{n+1}} ;
$$

i.e., for all $\varepsilon>0$ there exists a $\delta>0$ such that $\|z(\cdot, t)\|_{n}<\varepsilon$ for all $t>0$ and for all $z(\cdot, 0)$ such that $\|z(\cdot, 0)\|_{n}<\delta$.

Proof. We know that the perturbation $z(s, t)=\mathcal{O}(t)$. First we will show that there are no $\mathcal{O}(t)$ terms in $u_{1}(s, t)$ and $k(s, t)$ and that $\tilde{u}_{1}(\omega, t)$ and $\tilde{k}(\omega, t)$ are bounded in time for all $\omega$. However, the bounds are not uniform in $\omega$ so more precise estimates will be required to establish the final result.

First, we calculate the perturbations to the potential vorticity and true momentum:

$$
\begin{aligned}
j & =\left.\frac{\mathrm{d}}{\mathrm{d} \varepsilon}\right|_{\varepsilon=0} q_{s} \cdot p \\
& =\left.\frac{\mathrm{d}}{\mathrm{d} \varepsilon}\right|_{\varepsilon=0}\left(\begin{array}{c}
\varepsilon u_{1 s} \\
1+\varepsilon u_{2 s}
\end{array}\right) \cdot\left(\begin{array}{c}
1+\varepsilon v_{1} \\
\varepsilon v_{2}
\end{array}\right) \\
& =u_{1 s}+v_{2}, \\
k & =\left.\frac{\mathrm{d}}{\mathrm{d} \varepsilon}\right|_{\varepsilon=0} \frac{p(s, t)}{\left\|q_{s}(s, t)\right\|} \\
& =\left.\frac{\mathrm{d}}{\mathrm{d} \varepsilon}\right|_{\varepsilon=0} \frac{\left(\begin{array}{c}
1+\varepsilon v_{1} \\
\varepsilon v_{2}
\end{array}\right)}{\sqrt{\left.\left(\varepsilon u_{1 s}\right)^{2}+\left(1+\varepsilon u_{2 s}\right)^{2}\right)}} \\
& =\left(\begin{array}{c}
v_{1}-u_{2 s} \\
v_{2}
\end{array}\right) \cdot
\end{aligned}
$$

Of course, since $J$ is conserved by the nonlinear motion, $j$ is conserved by the linearized equations of motion. We express the linear system (3.1) in Jordan canonical form for $\omega \neq 0$ as

$$
\begin{aligned}
& \dot{y}_{1}=y_{2}, \\
& \dot{y}_{2}=0 \\
& \dot{y}_{3}=i \lambda y_{3}, \\
& \dot{y}_{4}=-i \lambda y_{4},
\end{aligned}
$$

where $\lambda=\sqrt{\left.\widetilde{G} \widetilde{F}\right|_{0} ^{\omega}(\mu-1)}$ and $\tilde{z}=X y$ with

$$
X=\left(\begin{array}{cccc}
0 & -a / c & -1 & -1 \\
1 & 0 & a \sqrt{\widetilde{G} / \beta} & -a \sqrt{\widetilde{G} / \beta} \\
-a & 0 & c / \sqrt{\widetilde{G} \beta} & -c / \sqrt{\widetilde{G} \beta} \\
0 & 1 / \widetilde{G} & -a & -a
\end{array}\right),
$$

where $\beta=c-(2 \pi \omega)^{2} \widetilde{G}$. As a check, we calculate that $y_{2}=(-a \widetilde{G} c / \beta)\left(-a \tilde{z}_{1}+\tilde{z}_{4}\right)$ which is proportional to the Fourier transform of $u_{1 s}+v_{2}$, the perturbed potential vorticity, which is 
why $\dot{y}_{2}=0$ (recall that $\left.a=-2 \pi i \omega\right)$. Therefore, the solution of (3.1) for $\omega \neq 0$ is

$$
\begin{aligned}
\widetilde{z} & =X y \\
& =X\left(\begin{array}{c}
y_{2}(0) t \\
0 \\
0 \\
0
\end{array}\right)+\mathcal{O}(1) \\
& =y_{2}(0) t\left(\begin{array}{c}
0 \\
1 \\
2 \pi i \omega \\
0
\end{array}\right)+\mathcal{O}(1),
\end{aligned}
$$

from which we conclude that $\tilde{u}_{1}, \tilde{v}_{2}$, and $\tilde{k}=\tilde{v}_{1}-2 \pi i \omega \tilde{u}_{2}$ are bounded in time for each $\omega$.

To study the motion in more detail we let $w=\left(u_{1 s}, u_{2}, k, j\right)^{T}=L z, \tilde{w}=\widetilde{L} \tilde{z}$, and compute the solution

$$
\tilde{w}(t)=\widetilde{L} X \operatorname{diag}\left(1,1, \mathrm{e}^{i \lambda t}, \mathrm{e}^{-i \lambda t}\right) X^{-1} \widetilde{L}^{-1} \tilde{w}(0),
$$

of which the relevant components are

$$
\begin{aligned}
\widetilde{u_{1 s}}(\omega, t) & =\cos (\lambda t) \widetilde{u_{1 s}}(\omega, 0)+\sin (\lambda t) \gamma(\omega) \tilde{k}(\omega, 0)-(1-\cos (\lambda t)) \gamma(\omega)^{2} \tilde{j}(\omega, 0), \\
\tilde{k}(\omega, t) & =\cos (\lambda t) \tilde{k}(\omega, 0)-\sin (\lambda t)\left(\gamma(\omega)^{-1} \widetilde{u_{1 s}}(\omega, 0)+\gamma(\omega) \tilde{j}(\omega, 0)\right),
\end{aligned}
$$

where

$$
\begin{aligned}
\gamma(\omega) & =2 \pi i \omega \sqrt{\widetilde{G} / \beta} \\
& =2 \pi \omega\left(\left(1+4 \pi^{2} \omega^{2}\right)^{1 / 2}-1\right)^{-1 / 2} \\
& = \begin{cases}\sqrt{2}, & \omega \rightarrow 0 \\
\mathcal{O}\left(\omega^{1 / 2}\right), & \omega \rightarrow \infty\end{cases}
\end{aligned}
$$

Using $\lambda(0)=0$ we can check that (3.29) holds for $\omega=0$ as well as for $\omega \neq 0$. Elementary calculus now establishes the bounds

$$
\begin{aligned}
|\gamma(\omega)| & \leq \sqrt{2 \pi}(1+|\omega|)^{1 / 2} \quad \forall \omega \\
\left|\gamma(\omega)^{-1}\right| & \leq \frac{1}{\sqrt{2}}(1+|\omega|)^{-1 / 2} \quad \forall \omega .
\end{aligned}
$$

We will also use

$$
\begin{aligned}
(1+|\omega|)^{l}\left(1+|\omega|^{2 n}\right) & \leq \sqrt{2}\left(1+|\omega|^{2 n+l}\right) \\
(1+|\omega|)^{-l}\left(1+|\omega|^{2 n}\right) & \leq\left(1+|\omega|^{2 n-l}\right)
\end{aligned}
$$


for all $\omega$ and for all $n, l>0$. We then have

$$
\begin{aligned}
\left\|u_{1 s}(\cdot, t)\right\|_{H^{n}} \leq & \left.\left\|u_{1 s}(\cdot, 0)\right\|_{H^{n}}+\left\|\mathcal{F}^{-1} \gamma(\omega) \tilde{k}(\omega, 0)\right\|_{H^{n}}+2 \| \mathcal{F}^{-1} \gamma(\omega)^{2}\right) \tilde{j}(\omega, 0) \|_{H^{n}} \\
= & \left\|u_{1 s}(\cdot, 0)\right\|_{H^{n}}+\left(\int(1+|\omega|)^{2 n}|\gamma(\omega) \tilde{k}(\omega, 0)|^{2} \mathrm{~d} \omega\right)^{1 / 2} \\
& +\left(\int(1+|\omega|)^{2 n}\left|\gamma(\omega)^{2} \tilde{j}(\omega, 0)\right|^{2} \mathrm{~d} \omega\right)^{1 / 2} \\
\leq & \left\|u_{1 s}(\cdot, 0)\right\|_{H^{n}}+\left(2 \sqrt{2} \pi \int\left(1+|\omega|^{2 n+1}\right)|\tilde{k}(\omega, 0)|^{2} \mathrm{~d} \omega\right)^{1 / 2} \\
& \quad+2\left(4 \sqrt{2} \pi^{2} \int\left(1+|\omega|^{2 n+2}\right)|\tilde{j}(\omega, 0)|^{2} \mathrm{~d} \omega\right)^{1 / 2} \\
= & \left\|u_{1 s}(\cdot, 0)\right\|_{H^{n}}+\sqrt{2 \sqrt{2} \pi}\|k(\cdot, 0)\|_{H^{n+1 / 2}}+2^{1 / 4} 4 \pi\|j(\cdot, 0)\|_{H^{n+1}}
\end{aligned}
$$

and similarly

$$
\begin{aligned}
\|k(\cdot, t)\|_{H^{n+1 / 2}} \leq & \|k(\cdot, 0)\|_{H^{n}+1 / 2}+\left\|\mathcal{F}^{-1} \gamma(\omega)^{-1} \widetilde{u_{1 s}}(t)\right\|_{H^{n+1 / 2}}+\left\|\mathcal{F}^{-1} \gamma(\omega) \tilde{j}(0)\right\|_{H^{n+1 / 2}} \\
= & \|k(\cdot, 0)\|_{H^{n}+1 / 2}+\left(\int(1+|\omega|)^{2 n+1}\left|\gamma(\omega)^{-1} \tilde{k}(\omega, 0)\right|^{2} \mathrm{~d} \omega\right)^{1 / 2} \\
& \quad+\left(\int(1+|\omega|)^{2 n+1}|\gamma(\omega) \tilde{j}(\omega, 0)|^{2} \mathrm{~d} \omega\right)^{1 / 2} \\
\leq & \|k(\cdot, 0)\|_{H^{n}+1 / 2}+\left(\frac{1}{2} \int\left(1+|\omega|^{2 n}\right)|\tilde{k}(\omega, 0)|^{2} \mathrm{~d} \omega\right)^{1 / 2} \\
& \quad+\left(2 \sqrt{2} \pi \int\left(1+|\omega|^{2 n+2}\right)|\tilde{j}(\omega, 0)|^{2} \mathrm{~d} \omega\right)^{1 / 2} \\
= & \left.\|k(\cdot, 0)\|_{H^{n+1 / 2}}+\frac{1}{\sqrt{2}}\left\|u_{1 s}(\cdot, 0)\right\|_{H^{n}}+\sqrt{2 \sqrt{2} \pi}\|j(\cdot, 0)\|\right)_{H^{n+1}} .
\end{aligned}
$$

Because $j$ is a conserved quantity, we also have that $\|j(\cdot, t)\|_{H^{n+1}}=\|j(\cdot, 0)\|_{H^{n+1}}$. Therefore

$$
\begin{aligned}
\|z(\cdot, t)\|_{n} & =\left\|u_{1 s}(\cdot, t)\right\|_{H^{n}}+\|k(\cdot, t)\|_{H^{n+1 / 2}}+\|j(\cdot, t)\|_{H^{n+1}} \\
& \leq\left(1+\frac{1}{\sqrt{2}}\right)\left\|u_{1 s}(\cdot, 0)\right\|_{H^{n}}+(1+\sqrt{2 \sqrt{2} \pi})\|k(\cdot, 0)\|_{H^{n+1 / 2}}+C\|j(\cdot, 0)\|_{H^{n+1}} \\
& \leq C\|z(\cdot, 0)\|_{n},
\end{aligned}
$$

where $C=1+\sqrt{2 \sqrt{2} \pi}+2^{1 / 4} 4 \pi$. That is, we have the required stability with $\delta=\varepsilon / C$.

Note that stability of the $y$-component $v_{2}$ of the true momentum follows from $v_{2}=j-u_{1 s}$, which implies that $\left\|v_{2}(\cdot, t)\right\|_{H^{n}} \leq\|j(\cdot, t)\|_{H^{n}}+\left\|u_{1 s}(\cdot, t)\right\|_{H^{n}} \leq C\|z(\cdot, 0)\|_{n}$ for some constant $C$.

It is necessary in Proposition 3.9 for the norm to include $u_{1 s}$ and not $u_{1}$ itself, the horizontal perturbation, for $u_{1}$ is not in fact bounded. To see why, we examine the $\omega=0$ Fourier mode of the solution. From (3.1), this obeys $\dot{\tilde{u}}(0, t)=\widetilde{G}(0) \tilde{v}(0, t), \dot{\tilde{v}}(0, t)=0$, with solution 
$\tilde{u}(0, t)=\tilde{u}(0,0)+\widetilde{G}(0) \tilde{v}(0,0) t, \tilde{v}(0, t)=\tilde{v}(0,0)$. In other words, the average speed of the sheet is increased by $\widetilde{G}(0) \int v(s, 0) \mathrm{d} s$, leading to a linear drift away from the unperturbed position. However, it can be proved, analogously to the proof of Proposition 3.9, that the motion is orbitally stable in a norm including $u_{1}(\cdot, 0)$.

By the Sobolev embedding theorem, these results also imply that the derivatives of the solution up to a suitable order are also bounded by $C \varepsilon$ for some constant $C$.

3.4. Numerical study of the instability. To follow the nonlinear development of the instability we will solve the Euler equations for momentum sheets, (1.8), numerically. The basic method is to discretize the sheet by a set of Lagrangian particles, or, equivalently, to approximate the integrals in (1.8) by constant-weight quadrature. Let the particles have positions $q_{i}$ and momenta $p_{i}, i=1, \ldots, N$, and let the particle spacing be $h$. The Hamiltonian becomes

$$
H=\frac{1}{2} h^{2} \sum_{i, j=1}^{N} G\left(\left\|q_{i}-q_{j}\right\|\right) p_{i} \cdot p_{j}
$$

and the equations of motion are

$$
\begin{aligned}
& \dot{q}_{i}=h \sum_{j=1}^{N} G\left(\left\|q_{i}-q_{j}\right\|\right) p_{j} \\
& \dot{p}_{i}=-h \sum_{j=1}^{N} p_{i} \cdot p_{j} G^{\prime}\left(\left\|q_{i}-q_{j}\right\|\right) \frac{q_{i}-q_{j}}{\left\|q_{i}-q_{j}\right\|} .
\end{aligned}
$$

We integrate these with the symplectic midpoint rule $[4,18] x_{k+1}=x_{k}+\Delta t f\left(\bar{x}_{k}\right)$, where the midpoint value $\bar{x}_{k}=\left(x_{k}+x_{k+1}\right) / 2$. Previous midpoint values are stored and extrapolated to provide a high-order initial guess for $\bar{x}_{k}$, so that one can solve for $x_{k+1}$ by simple iteration to within the desired tolerance (typically $10^{-12}$ ) in just two or three iterations. Higher-order variants were tested but not needed.

To simulate periodic sheets (say, with period 1 in the $y$-direction), it is necessary to augment the Hamiltonian to include the effect of the periodic images:

$$
H_{\mathrm{ext}}=\frac{1}{2} h^{2} \sum_{i, j=1}^{N} \sum_{k=-M}^{M} G\left(\left\|q_{i}-q_{j}+k(0,1)^{T}\right\|\right) p_{i} \cdot p_{j} .
$$

The parameter $M$ (the number of periodic images) is adjusted so that the contribution of the omitted images is less than $10^{-12}$. Since $G(r)=o\left(e^{-y / \alpha}\right)$ in all the cases we consider, and we use a length-scale $\alpha=0.2, M \leq 3$ is sufficient. (In the motion of vortex sheets, governed by the Biot-Savart law, the contribution of all the periodic images can be summed analytically; but we could not achieve this for our choice of kernel.)

For analytic circular (resp., periodic) sheets and analytic $G$, the discretization (3.36) (resp., (3.38)) corresponds to discretizing an analytic periodic integral by the trapezoidal rule and hence is spectrally accurate. For $H^{k}$ metrics, however, $G$ is not analytic. In this case we apply the theory of quadrature of weakly singular periodic integrands developed by Sidi and Israeli [26]. (A similar issue arises for axisymmetric vortex sheets [23].) 
Theorem 3.10 (from [26]). If $\phi(u)=|u-s|^{\sigma} \log |u-s| f(u)+\tilde{f}(u), \sigma>-1$, with $f(u)$ and $\tilde{f}(u) 2 m$ times differentiable on $[a, b]$, and $\phi(u)$ periodic with period $T=b-a$ and $2 m$ times differentiable on $(-\infty, \infty) \backslash\{t+k T\}_{k=-\infty}^{\infty}$, grid points $u_{j}=a+j h, h=(b-a) / n, s=u_{i}$ for some $i, \zeta$ the Riemann zeta function, and

$$
Q_{n}[\phi]=h \sum_{\substack{j=1 \\ u_{j} \neq s}}^{n} \phi\left(u_{j}\right)+\tilde{f}(s) h+2\left[\zeta^{\prime}(-\sigma)-\zeta(-\sigma) \log h\right] f(s) h^{\sigma+1}
$$

then the error

$$
\int_{a}^{b} \phi(u) \mathrm{d} u-Q_{n}[\phi]=2 \sum_{\mu=1}^{m-1}\left[\zeta^{\prime}(-\sigma-2 \mu)-\zeta(-\sigma-2 \mu) \log h\right] \frac{f^{(2 \mu)}(s)}{(2 \mu) !} h^{2 \mu+\sigma+1}+\mathcal{O}\left(h^{2 m}\right)
$$

as $h \rightarrow 0$.

Corollary 3.11. The Hamiltonians (3.36) for circular sheets and (3.38) for straight sheets, for Euclidean-invariant $H^{k}$ metrics with $k \geq 2$, have quadrature errors of $\mathcal{O}\left(h^{2 k-1}\right)$.

Proof. The form of the singularity in $G(r)$ at $r=0$ is controlled by the highest derivatives $\nabla^{2 k}$ in the inertia operator $\mathcal{A}$ of the metric. $G(r)$ has the form used in Theorem 3.10 with $\sigma=2 k-2$. For $k \geq 2$, the term $\tilde{g}(t)=G(0)$ is included in the quadrature in (3.36); the term $\zeta(-\sigma)$ drops out because $\zeta(2-2 k)=0$ for $k \geq 2$; but the term $\zeta^{\prime}(-\sigma)$ is missing from the quadrature (3.36) and hence determines the leading order error of the quadrature. For circular sheets, the integrands are periodic as in Theorem 3.10, while for straight sheets the integrands decay rapidly at infinity, so there are no boundary terms.

The interesting case $k=1$ requires more attention. In this case $G(r) \sim c \log |r|$ as $r \rightarrow 0$, i.e., $\sigma=0$ in (3.39). Simply omitting the singular term from the integrand, i.e., setting $G(0)=0$, gives a very poor quadrature rule of error $\mathcal{O}(h \log h)$. Consider the integrand $G(\|q(s)-q(u)\|) p(u)$, for which an expansion near $u=s$ gives

$$
G(\|q(s)-q(u)\|) p(u)=c \log \left(|s-u|\left\|q^{\prime}(s)\right\|\right) p(s)+o(s-u),
$$

i.e., $f(s)=c p(s)$ and $\tilde{f}(s)=c \log \left\|q^{\prime}(s)\right\| p(s)$. We could therefore apply the quadrature of (3.39) to get a method of order $\mathcal{O}\left(h^{3}\right)$. However, this would involve approximating the term $\left\|q^{\prime}(s)\right\|$ by, e.g., finite differences, leading to complicated ODEs. Instead, we have started with the quadrature

$$
P_{n}[\phi]=-2 c p(s) \zeta(0) h \log h+h \sum_{\substack{j=1 \\ u_{j} \neq s}}^{n} \phi\left(u_{j}\right)
$$

which is $\mathcal{O}(h)+\mathcal{O}\left(h^{3}\right)$ accurate. It can be interpreted as a regularization of the kernel with $G(0)=-2 c \zeta(0) \log h=c \log h$. One step of Richardson extrapolation, i.e., $2 P_{n}[\phi]-P_{n / 2}[\phi]$, eliminates the $\mathcal{O}(h)$ term, establishing the following. 
Proposition 3.12. For kernels obeying $G(r) \sim c \log |r|$ as $r \rightarrow 0$, the quadrature error

$$
\int G(\|q(s)-q(u)\|) p(u) \mathrm{d} u-h \sum_{j=1}^{n} G_{i j} p_{j}=\mathcal{O}\left(h^{3}\right)
$$

where

$$
G_{i j}= \begin{cases}G\left(\left\|q_{i}-q_{j}\right\|\right), & j-i \text { odd } \\ -2 c \log 2, & j=i, \\ 0, & j \neq i, j-i \text { even } .\end{cases}
$$

The quadrature here is essentially the "every other point" trapezoidal rule (as studied, e.g., as the modified point vortex method in [3]) but with the singular point included with a special weight to account for the singularity.

For the standard $H^{1}$ metric with $\mathcal{A}=\left(1-\alpha^{2} \nabla^{2}\right)$, we have $G(r)=K_{0}(r) /\left(2 \pi \alpha^{2}\right) \sim$ $-\frac{1}{2 \pi \alpha^{2}} \log |r|$ as $r \rightarrow 0$, i.e., $c=-\frac{1}{2 \pi \alpha^{2}}$.

There are two further numerical difficulties with the $H^{1}$ kernel. First, recall from section 3.2 that the bounded growth rates of the high frequencies depend delicately on the asymptotic properties of $\widetilde{G}(\omega)$. These properties are destroyed by the numerical quadrature of (3.42), and indeed, a direct solution of Hamilton's equations shows a high frequency instability. We have suppressed this instability using the Krasny filter [13]; during the simulation, any Fourier modes with an amplitude less than a chosen cutoff (we used $10^{-10}$ ) are set to 0 . This prevents growth of the instability until the amplitudes of all modes are greater than the cutoff. After this time the evolution of the sheet tends to proceed very quickly until it reaches the point at which all accuracy is lost, typically just before a collision of the sheet with itself, so that the instability does not have time to dominate the solution. Convergence of point vortex methods for vortex sheets using the Krasny filter is established in [3].

The second difficulty occurs only in the simulation of sheets moving transversely to themselves, i.e., in the case $\phi=0$. Recall that this case has a double zero eigenvalue for each wavenumber. Under the numerical discretization, this double zero splits into a \pm pair of eigenvalues of the order of the square root of the discretization error. This is not a problem for the Gaussian kernel, for which we have spectral accuracy, but it is a problem for the $H^{1}$ case. The eigenvalue pair can be real or imaginary depending on $h$ and on the metric. If real, it creates a numerical instability affecting all wavenumbers. The only way we have found to deal with this is to perform careful convergence checks of all our results for this case.

The results for a number of simulations are shown in Figures 4-11 along with some error estimates. (There are animations of the sheet motion available for each of these cases at http://www-ist.massey.ac.nz/smarsland/KelvinHelmholtz.html.) In each case we have computed three approximate solutions, $x^{(1)}=x_{h, \Delta t}, x^{(2)}=x_{\frac{1}{2} h, \frac{1}{2} \Delta t}$, and $x^{(3)}=x_{h, \frac{1}{2} \Delta t}$. The global error in $x^{(2)}$ due to the (second-order) time discretization is estimated by $\left(x^{(3)}-x^{(1)}\right) / 3$. The estimation of the global error due to the space discretization depends on the metric. For the $H^{1}$ metric, it is estimated for $x^{(2)}$ by $\left(x^{(2)}-x^{(3)}\right) / 7$ (since the spatial error is $\mathcal{O}\left(h^{3}\right)$ ), and for the Gaussian metric it is estimated for (the less accurate solution) $x^{(3)}$ by $x^{(2)}-x^{(3)}$; the error in $x^{(2)}$ is much smaller. We also compute the error in the potential vorticity $J(s)$. We then take 2-norms with respect to space for all the errors and plot their evolution in time 


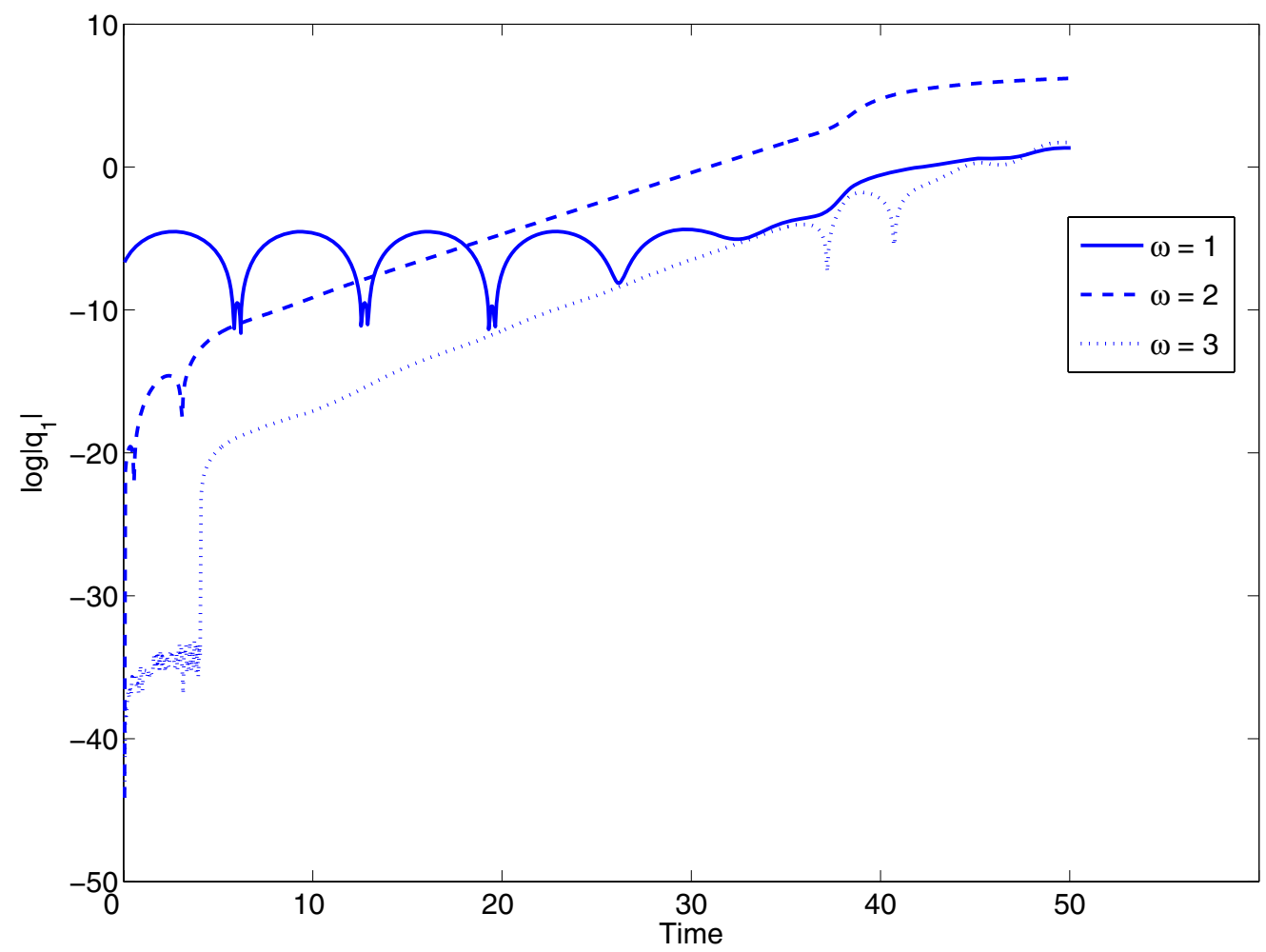

Figure 4. Evolution of an initially nearly straight momentum sheet under a Gaussian metric with unperturbed motion normal to the sheet $(\phi=0)$. The initial perturbation is confined to the stable mode $\omega=1$, which oscillates while triggering a nonlinear response in the most unstable mode $\omega=2$, which entrains a forced response in the less unstable mode $\omega=3$.
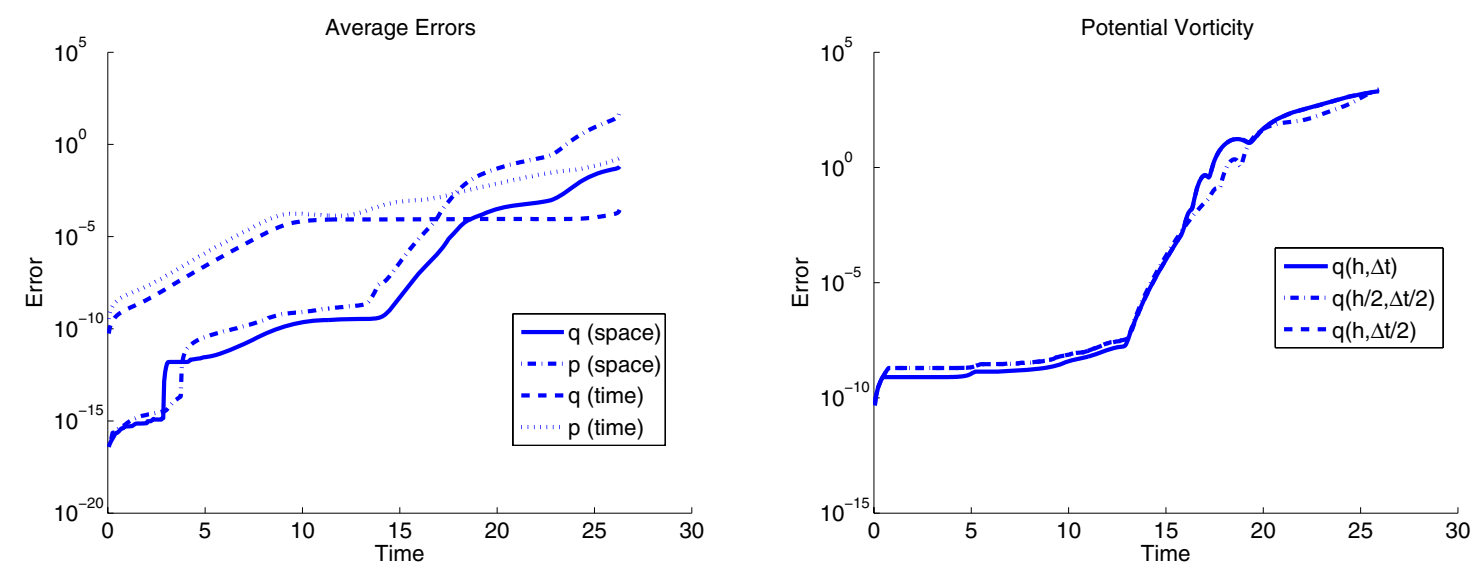

Figure 5. The spatial and temporal components of the mean global error in $q$ and $p$ (left) and the error in a pseudospectral estimate of the potential vorticity (right) for the simulation of Figure 4. 

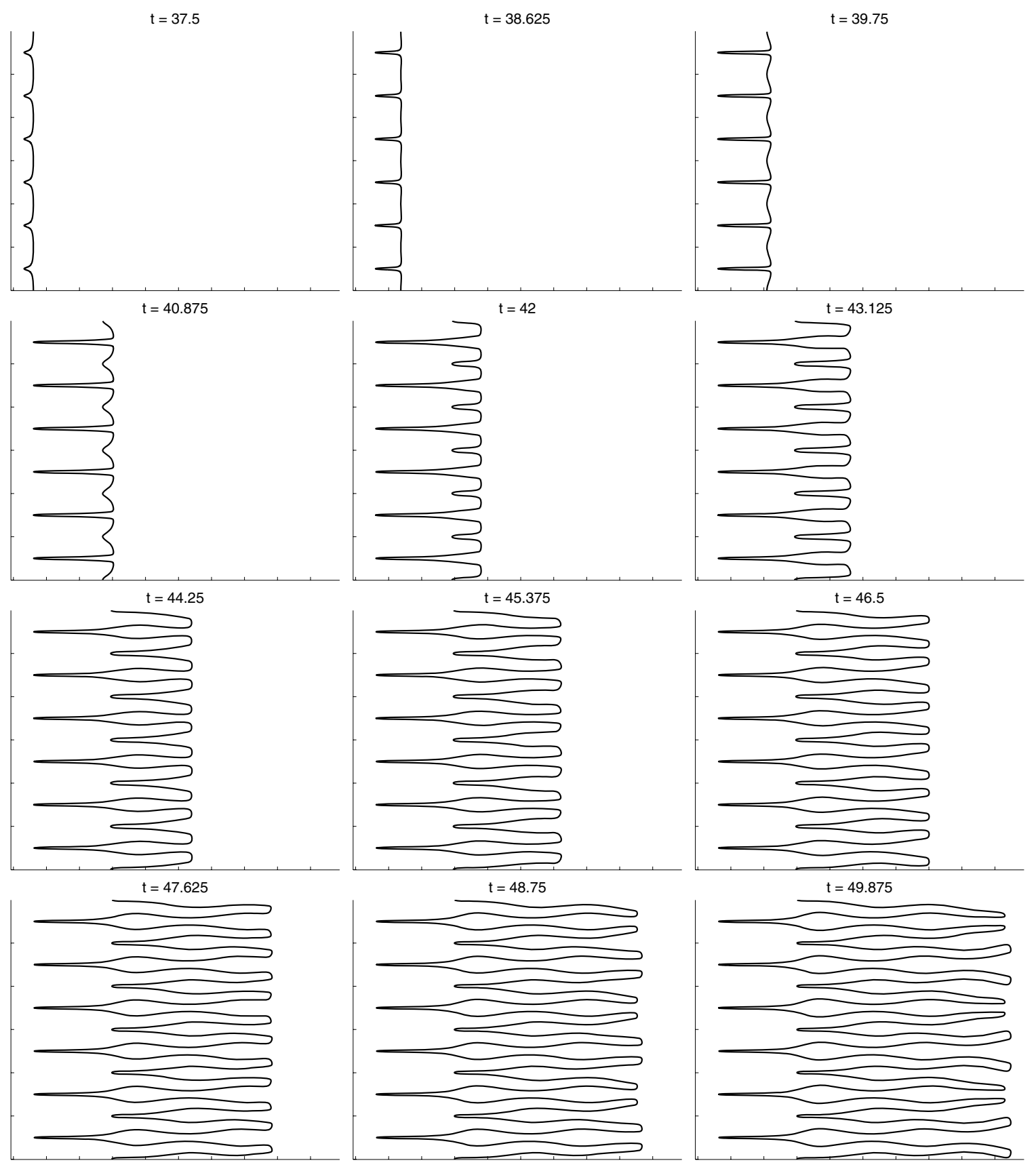

Figure 6. The motion of the sheet for the simulation of Figure 4, i.e., Gaussian metric and $\phi=0$. The solutions are 1-periodic in the $y$-direction and three symmetric images are shown. See text for further details. The $x$ and $y$ axes have the same scaling throughout.

for the Gaussian kernel in Figure 5. In all cases $h=1 / 512$ and $\Delta t=0.05$, so that for the most accurate solution $x^{(2)}$ there are 1024 grid points and the time step is 0.025. (In fact, the dominant errors can be removed by Richardson extrapolation applied to the final results; this 


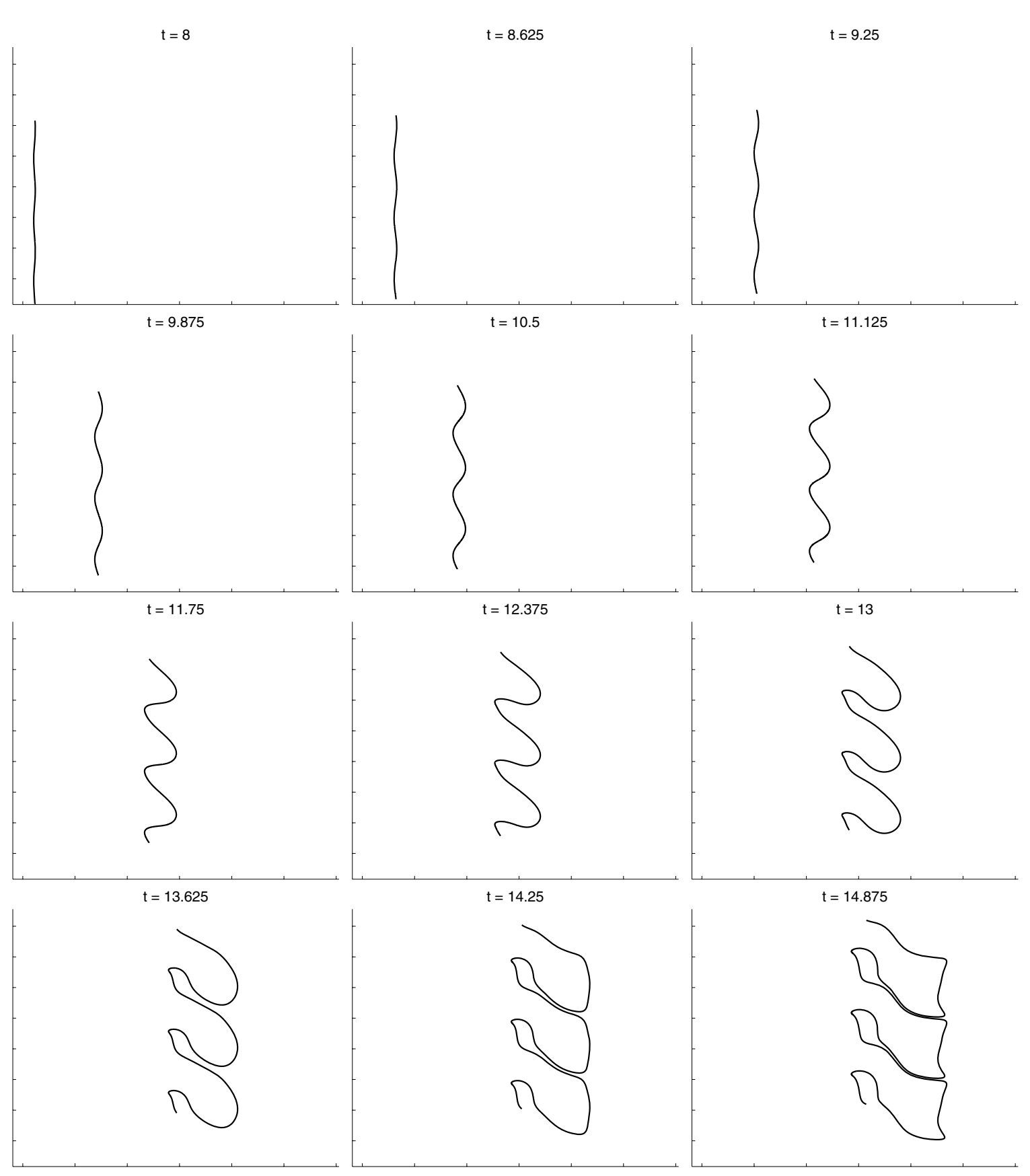

Figure 7. The motion of the sheet for the Gaussian metric and $\phi=\pi / 8$.

does not change our observations of the results.)

For the major part of all the simulations the errors are reasonably small. However, when the sheets become stretched or develop sharp corners, the errors are large and the results should be interpreted with caution. In addition, it is known [10] that the point particle 


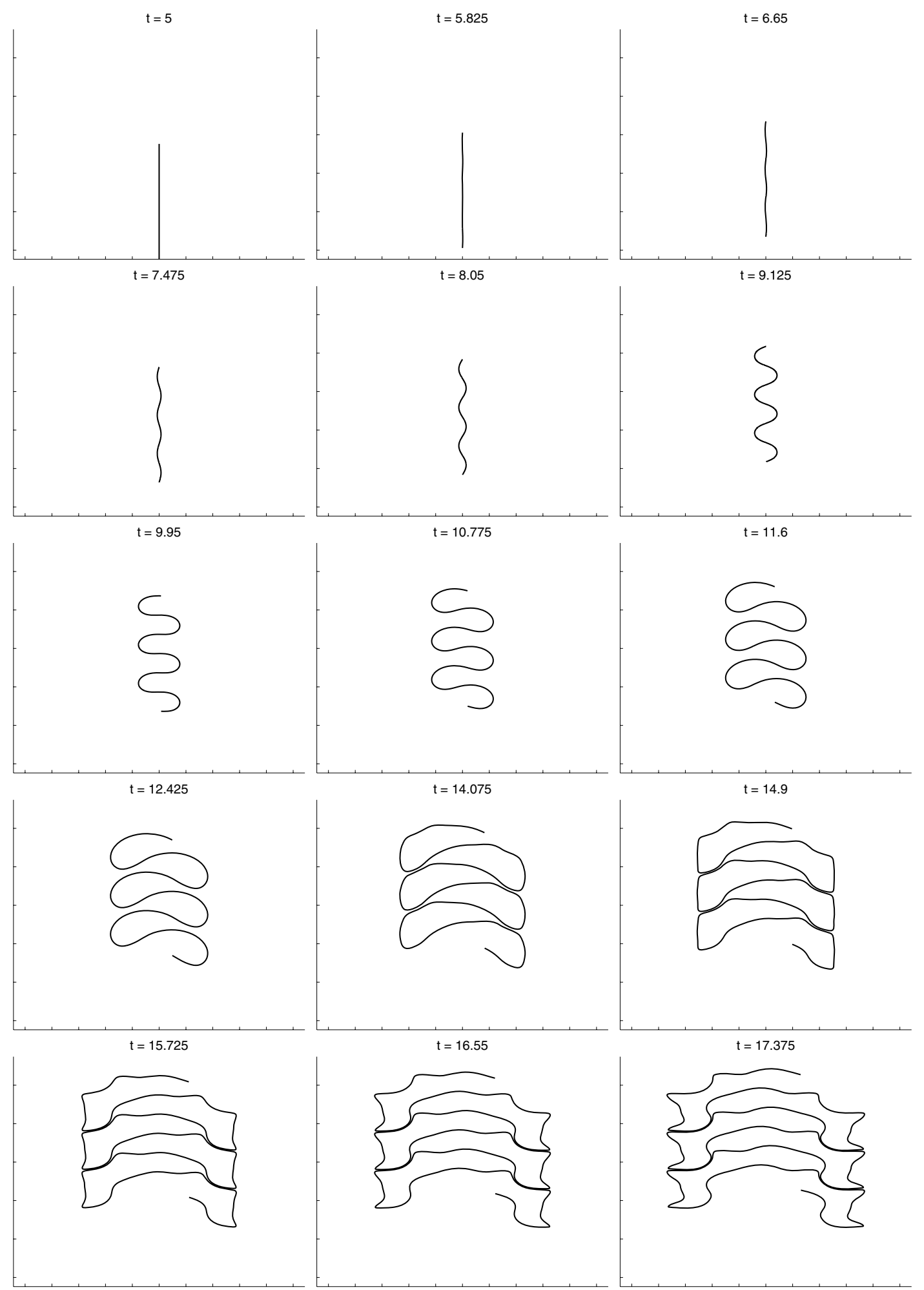

Figure 8. The motion of the sheet for the Gaussian metric and $\phi=\pi / 2$. 

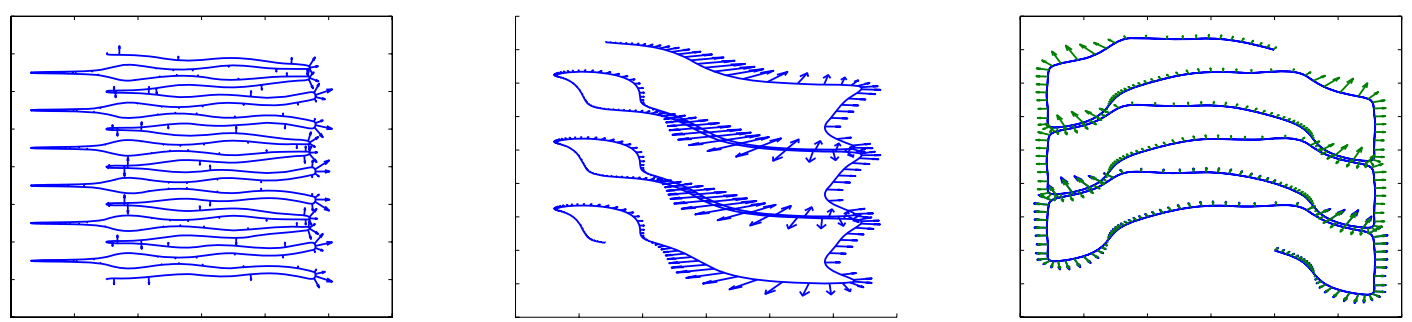

Figure 9. Close ups of the motion for the Gaussian metric, showing the momentum (at every 10th point). Left: $\phi=0$; see Figure 6. Center: $\phi=\pi / 8$; see Figure 7 . Right: $\phi=\pi / 2$; see Figure 8.

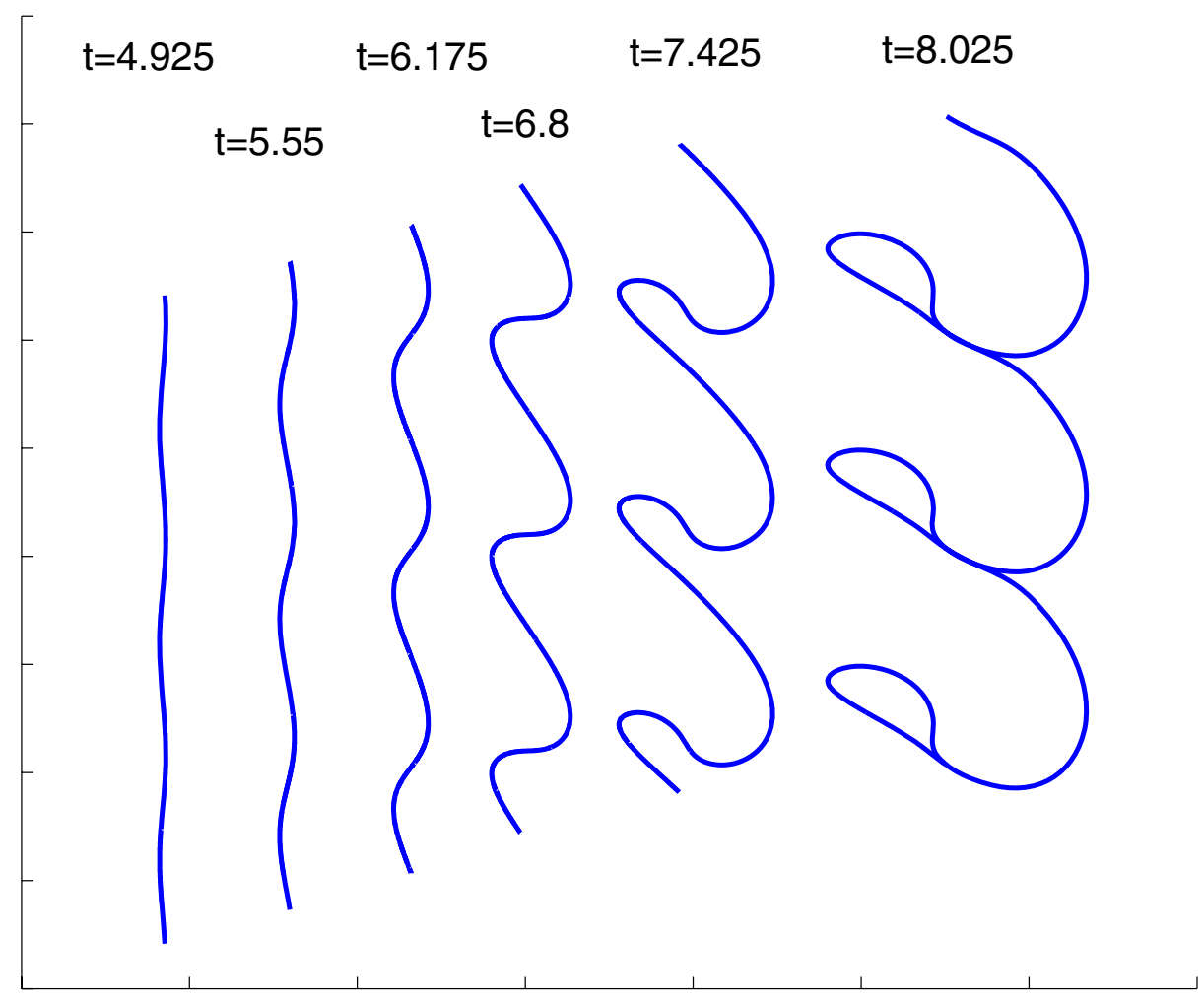

Figure 10. The motion of the sheet for the $H^{1}$ metric and $\phi=\pi / 8$.

approximation for sheets becomes inaccurate when two sections of the sheet approach closer than a few times the particle spacing.

For the Gaussian kernel, the numerical results indicate that the sheet remains analytic for all time and does not develop spontaneous singularities or self-intersections. For the $H^{1}$ kernel, 


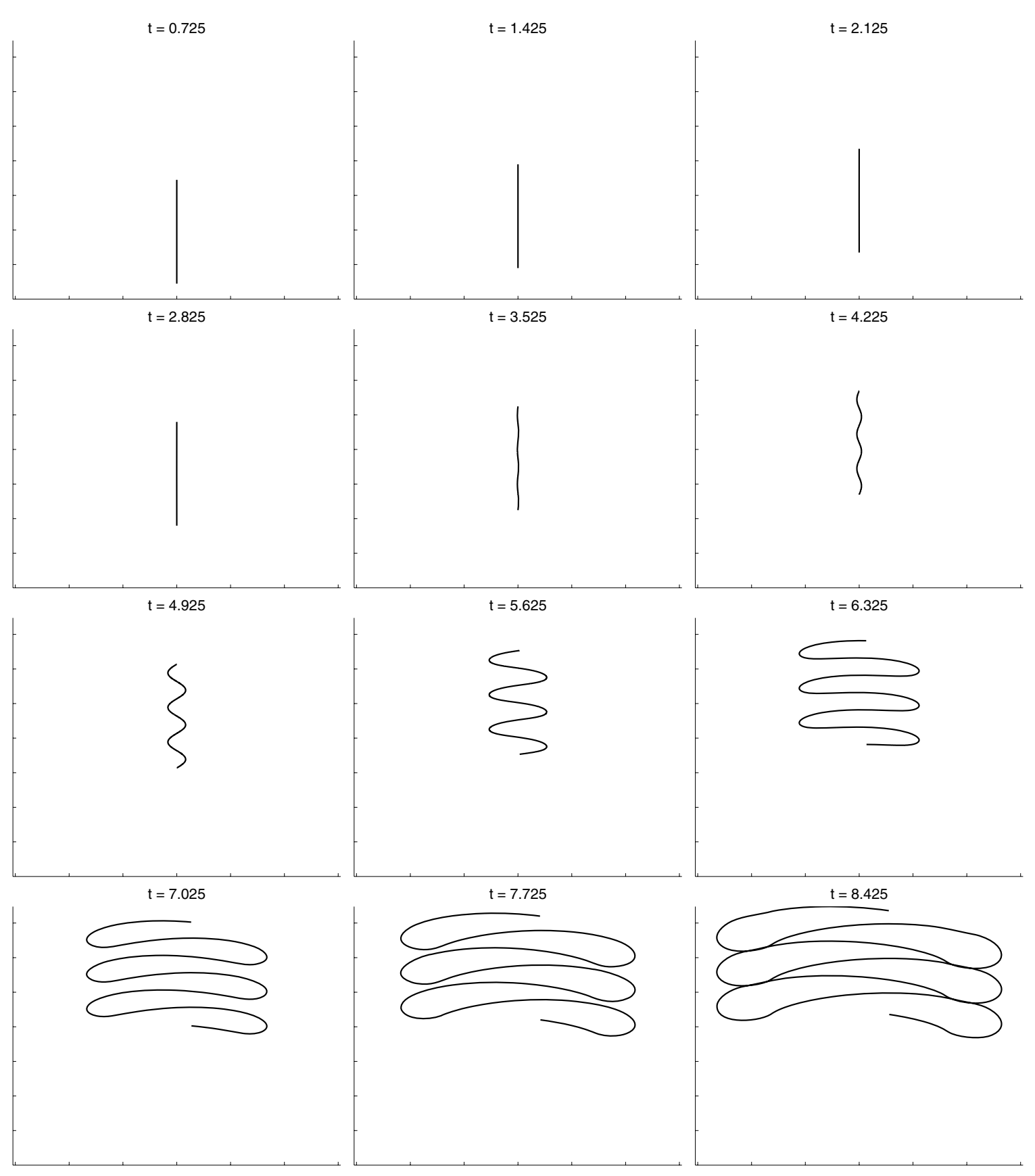

Figure 11. The motion of the sheet for the $H^{1}$ metric and $\phi=\pi / 2$.

the motion can terminate in a finite-time self-intersection. Indeed, this is already known to happen when two 1D Camassa-Holm solitons approach one another, which corresponds here to the collision of two approaching straight sheets. The numerical results indicate, however, that the sheet remains analytic prior to a self-intersection and does not develop spontaneous 
self-intersections. In all cases the initial perturbation is confined to wavenumber 1 and has amplitude $5 \times 10^{-6}$ in $q$ and $2.5 \times 10^{-5}$ in $p$.

Three cases are shown for the Gaussian kernel $G(r)=\mathrm{e}^{-(r / \alpha)^{2}}$ with $\alpha=0.2$. (Note the different space- and time-scales from those considered in section 3.2. Note also that three symmetric repetitions of the sheet are shown.) For normal motion $(\phi=0)$, from section 3.2, we have that wavenumbers with $\alpha \omega>0.3568$ are unstable, with the instability strongly peaked at $\alpha \omega=0.4671$. In the simulations with periodic boundary conditions, only integer wavenumbers are permitted. Therefore, $\omega=1$ is stable, and $\omega \geq 2$ is unstable, with $\omega=2$ the most unstable. These results are confirmed in Figure 4, which shows wavenumber $\omega=1$ undergoing three periodic oscillations before the nonlinear interactions it generates in $\omega=2$ grow to dominate the solution. $\omega=3$ is slaved to the most rapidly growing mode $\omega=2$. The nonlinear evolution of the instability (Figure 6) shows the development of two fingers on length-scale $\alpha$, the tail of which stays put. The body of the finger consists of two sheets with opposite momentum, which approach each other like the capture of 1D particles [17] and essentially cancel one another out. This is the simplest instance of what we call the "zippering" phenomenon of sheets. The remaining straight part of the sheet then undergoes a second instability, also with $\omega=2$ but displaced by a quarter period. As these secondary fingers grow they cause the primary fingers to unzip. In the final state shown the motion consists of approximately 1D motion of the horizontal fingers combined with continued evolution of the tips.

For small nonzero $\phi$ the picture is qualitatively the same, but as $\phi$ increases, new features of the motion emerge. The motion for $\phi=\pi / 8$ is shown in Figure 7 . Wavenumber 1 is now unstable, and, in addition, the momentum in the tail of the finger swings around to form an outwardly expanding bubble. The zippered sections of the sheet are now curved and undergo dynamics of their own. Eventually the bubbles and zippers will meet their symmetric images and undergo further zipperings. The secondary instability also occurs but its evolution appears to be constrained by the primary zippers.

As $\phi$ increases further the bubbles grow larger, until the extreme case (Figure 8) $\phi=\pi / 2$ is reached. Now the bubbles are so large that there is no recognizable finger and no zippering at the bubble's tail. Instead, each left- and right-moving bubble expands to meet its periodic image, sections of these pairs zipper up, and the heads of each bubble in a beautiful maneuver reform to form left- and right-moving straight sheets. These, of course, are unstable and begin to finger, causing the main zippers to begin to unzip, while the partly zippered internal parts of the sheet continue to evolve in their own right.

The initial stages of the evolution of the nonlinear stability for the $H^{1}$ metric are roughly similar. (See Figure 10 for $\phi=\pi / 8$ and Figure 10 for $\phi=\pi / 2$.) We know, however, from the $1 \mathrm{D}$ case, that sheets can collide in finite time, with the classical solution ending in a singularity. All the simulations do appear to end in this way, although we have not performed a detailed study of the collision process. Intriguingly, however, when two sections of the sheet appear to be on a collision path, they first match their shapes and try to zipper up. Only after a long zippered section has formed do the sheets collide at some point on the zipper. In the case $\phi=\pi / 2$ the zippered section even appears to reseparate along its length before colliding at the ends of the zipper. Although these late-stage phenomena are observed in both the high- and low-accuracy simulations, they should still be interpreted with caution as the global errors here are large. 
4. Stability of expanding circular sheets. A single circular sheet is described by its radius $r(t)$, radial momentum $p_{r}(t)$, and rotational momentum $p_{\phi}(t)$. If the momentum points toward the center of the circle, the circle collapses to a point, ending in a singularity; otherwise the momentum vector is asymptotically outwardly normal to the sheet. Here we study only this limiting case; i.e., we take $p_{\phi}=0$.

The linearized equations of motion form a complicated system of integro-differential equations which, as far as we can tell, cannot be solved analytically. However, they can be solved asymptotically for large $r$ (and hence for large $t$ ). The following proposition shows that expanding circular sheets are algebraically, but not exponentially, unstable. Moreover, the instability does not depend in a significant way on the initial perturbation, which enters the solution only parametrically. All frequencies grow at the same rate. The growth relative to the size of the expanding sheet is only $\mathcal{O}\left(t^{1 / 3}\right)$.

Proposition 4.1. Consider a circular momentum sheet of radius $r(t)$, radial momentum $p_{r}(t)$, and rotational momentum $p_{\phi}(t)=0$ subject to a small perturbation, so that its position and momentum are described by

$$
q(r, t)=r(t)\left(\begin{array}{c}
\cos \theta \\
\sin \theta
\end{array}\right)+\varepsilon u(\theta, t), \quad p(r, t)=p_{r}(t)\left(\begin{array}{c}
\cos \theta \\
\sin \theta
\end{array}\right)+\varepsilon v(\theta, t),
$$

where $\theta$ is the Lagrangian parameter of the sheet. If $p_{r}(0)>0$ so that the sheet is expanding, then as $t \rightarrow \infty$ we have

$$
r(t)=\mathcal{O}\left(t^{2 / 3}\right), \quad p_{r}(t)=\mathcal{O}\left(t^{1 / 3}\right), \quad u(t)=\mathcal{O}(t), \quad v(t)=\mathcal{O}\left(t^{2 / 3}\right)
$$

for all kernels $G(r)$.

Proof. For fixed $\theta \neq \alpha$, the distance $d=\|q(\theta)-q(\alpha)\|_{2}$ between two points on the sheet is given by

$$
\begin{array}{rl}
d=2 & r \sin \frac{1}{2}|\alpha-\theta| \\
& +\varepsilon\left(2 \sin \frac{1}{2}|\alpha-\theta|\right)^{-1}\left[\left(v_{1}(\theta)-v_{1}(\alpha)\right)(\cos \theta-\cos \alpha)+\left(v_{2}(\theta)-v_{2}(\alpha)\right)(\sin \theta-\sin \alpha)\right] \\
& +\mathcal{O}\left(\varepsilon^{2}\right)
\end{array}
$$

The unperturbed motion is described asymptotically for large $r$ by a Hamiltonian system with $H=\left(G_{0} p_{r}^{2}\right) /(2 r)+\mathcal{O}\left(p_{r}^{2} / r^{3}\right)$, where $G_{0}=\int_{-\infty}^{\infty} G(r) \mathrm{d} r$; dropping the higher-order terms, the equations of motion are

$$
\begin{aligned}
\dot{r} & =G_{0} \frac{p_{r}}{r}, \\
\dot{p}_{r} & =-\frac{1}{2} G_{0} \frac{p^{2}}{r^{2}}
\end{aligned}
$$

with solution

$$
\begin{aligned}
r(t) & =\left(\frac{3}{2} \sqrt{2 G_{0} E} t+r_{0}^{3 / 2}\right)^{2 / 3}=\mathcal{O}\left(t^{2 / 3}\right), \\
p_{r}(t) & =\sqrt{2 E r(t) / G_{0}}=\mathcal{O}\left(t^{1 / 3}\right),
\end{aligned}
$$


where $E$ is the value of the energy. Note that the momentum per unit length of the sheet is $\mathcal{O}\left(t^{-1 / 3}\right)$ and tends to zero as $t \rightarrow \infty$.

Linearizing the equations of motion, making the substitution $\tilde{r}=2 r \sin \frac{1}{2}|\alpha-\theta|$, and expanding the integrands for large $r$ give the linearized system

$$
\begin{aligned}
& u_{t}=\frac{G_{0}}{r} v+\frac{G_{0} p_{r}}{r^{2}} A^{T}(\theta) u_{\theta}, \\
& v_{t}=\frac{G_{0} p_{r}}{r^{2}}(A(\theta) v)_{\theta}
\end{aligned}
$$

with coefficient matrix

$$
A(\theta)=\left(\begin{array}{cc}
\cos \theta \sin \theta & \sin ^{2} \theta \\
-\cos ^{2} \theta & -\cos \theta \sin \theta
\end{array}\right) .
$$

Equation (4.5) forms a canonical Hamiltonian system with Hamiltonian

$$
H=\int_{0}^{2 \pi}\left(\frac{G_{0} p_{r}}{r^{2}} u_{\theta}^{T} A v+\frac{G_{0}}{2 r} v^{T} v\right) \mathrm{d} \theta
$$

which can also be derived by directly expanding the full Hamiltonian. In general, of course, such nonautonomous, nonconstant-coefficient PDEs cannot be solved explicitly. In this case, however, the matrix $A$ has constant Jordan form,

$$
A=X J X^{-1}, \quad X=\left(\begin{array}{cc}
-\tan \theta & -\sec ^{2} \theta \\
1 & 0
\end{array}\right), \quad J=\left(\begin{array}{ll}
0 & 1 \\
0 & 0
\end{array}\right),
$$

and, even though $A(\theta)$ and $A(\alpha)$ do not commute for $\theta \neq \alpha$, the equations are nevertheless dramatically simplified by the canonical change of variables

$$
v=X \bar{v}, \quad u=X^{-T} \bar{u}
$$

in terms of which the new Hamiltonian is

$$
\begin{aligned}
\bar{H} & =\int_{0}^{2 \pi} \bar{u}^{T}\left(X^{-1} A X\right) \bar{v}+\bar{u}^{T}\left(X^{-1}\right)_{\theta} A X \bar{v}+\frac{1}{2} \bar{v}^{T} X^{T} X \bar{v} \mathrm{~d} \theta \\
& =\int_{0}^{2 \pi} \frac{G_{0} p_{r}}{r^{2}}\left(\bar{u}_{\theta}^{T} J v+\bar{u}^{T} K \bar{v}\right)+\frac{G_{0}}{2 r} \bar{v}^{T} L v \mathrm{~d} \theta,
\end{aligned}
$$

where

$$
K=\left(\begin{array}{cc}
0 & 0 \\
0 & -1
\end{array}\right), \quad L=\sec ^{2} \theta\left(\begin{array}{cc}
1 & \tan \theta \\
\tan \theta & \sec ^{2} \theta
\end{array}\right)
$$

with equations of motion

$$
\begin{aligned}
& \dot{\bar{u}}_{1}=\frac{G_{0}}{r} \sec ^{2} \theta\left(\bar{v}_{1}+\bar{v}_{2} \tan \theta\right) \\
& \dot{\bar{u}}_{2}=\frac{G_{0} p_{r}}{r^{2}}\left(\bar{u}_{1 \theta}-\bar{u}_{2}\right)+\frac{G_{0}}{r} \sec ^{2} \theta\left(\bar{v}_{1} \tan \theta+\bar{v}_{2} \sec ^{2} \theta\right) \\
& \dot{\bar{v}}_{1}=\frac{G_{0} p_{r}}{r^{2}} \bar{v}_{2 \theta} \\
& \dot{\bar{v}}_{2}=\frac{G_{0} p_{r}}{r^{2}} \bar{v}_{2}
\end{aligned}
$$




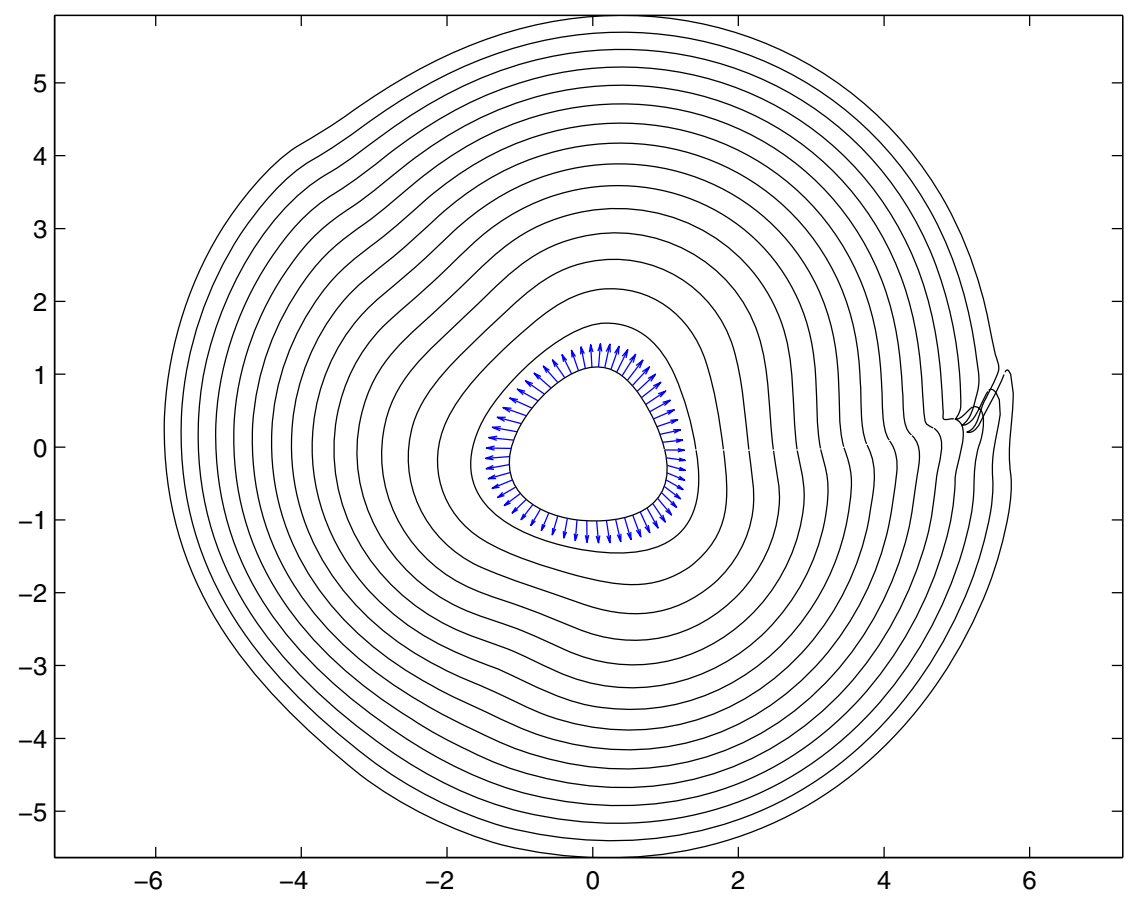

Figure 12. The nonlinear development of the algebraic $\left(\mathcal{O}\left(t^{1 / 3}\right)\right)$ instability of circular momentum sheets. Here $r(0)=p_{r}(0)=1, G(r)=\exp \left(-(r / 0.2)^{2}\right)$, and a small perturbation is applied to $q$ and $p$ in the 2 nd Fourier mode.

Notice that the change of variables has reduced the PDEs to (effectively) ODEs. Substituting the asymptotic behavior of $r(t)$ and $p_{r}(t)$ gives

$$
\begin{aligned}
& \dot{\bar{u}}_{1}=c t^{-2 / 3} \sec ^{2} \theta\left(\bar{v}_{1}+\bar{v}_{2} \tan \theta\right), \\
& \dot{\bar{u}}_{2}=\frac{2}{3 t}\left(\bar{u}_{\theta 1}-\bar{u}_{2}\right)+c t^{-2 / 3} \sec ^{2} \theta\left(\bar{v}_{1} \tan \theta+\bar{v}_{2} \sec ^{2} \theta\right), \\
& \dot{\bar{v}}_{1}=\frac{2}{3 t} \bar{v}_{2 \theta}, \\
& \dot{\bar{v}}_{2}=\frac{2}{3 t} \bar{v}_{2} .
\end{aligned}
$$

The solution for the momentum perturbation (as $t \rightarrow \infty)$ is

$$
\bar{v}_{2}=t^{2 / 3} b(\theta), \quad \bar{v}_{1}=t^{2 / 3} b^{\prime}(\theta),
$$

where $b(\theta)$ is determined by the initial conditions. Substituting (4.14) into (4.13) for $\bar{u}$ shows that only the $\mathcal{O}\left(t^{-2 / 3}\right)$ terms contribute to the leading order behavior of $\bar{u}$. This leading order behavior is more easily determined from the original formulation

$$
u_{t}=c t^{-2 / 3} v=\alpha d(\theta) \quad\left(\text { where } d=X\left(b^{\prime}, b\right)^{T}\right),
$$



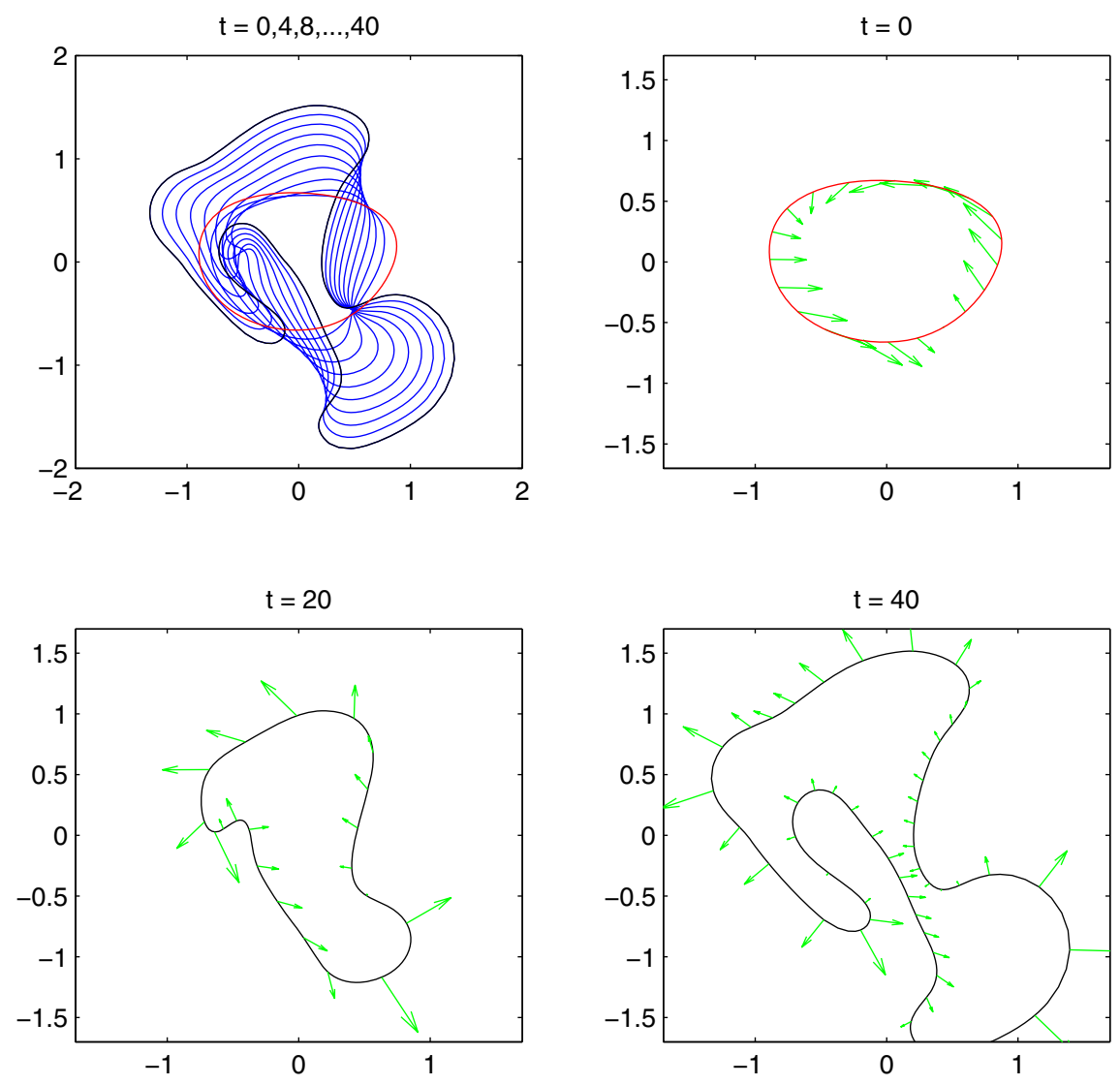

Figure 13. Motion of a momentum sheet with noncircular initial conditions, Gaussian metric.

where $\alpha=\left[\left(4 p_{r 0}^{2}\right) /\left(9 G_{0} r_{0}\right)\right]^{-1 / 3}$, so

$$
u=\gamma t d(\theta)
$$

Note that the asymptotic behavior of the unperturbed sheet and of the leading order behavior of perturbation depends on the metric only through $G_{0}$. Higher-order terms depend on higher moments of $G$, and these can be found through a regular perturbation expansion proceeding in powers of $r^{-2}$. Also, note that the $\mathcal{O}\left(t^{2 / 3}\right)$ growth of the sheet is critical in determining its stability; any slower, and the growth of perturbations would be bounded; any faster, and they would grow exponentially, not algebraically.

The development of the instability is shown in Figure 12 under the Gaussian metric $G(r)=\exp \left(-(r / \alpha)^{2}\right)$ with $\alpha=0.2$. Here $r(0)=p_{r}(0)=1$ and the initial perturbation is confined to the second Fourier mode. The nonlinear development of the instability shows itself on the length-scale $\alpha$, followed by rapid fingering. 

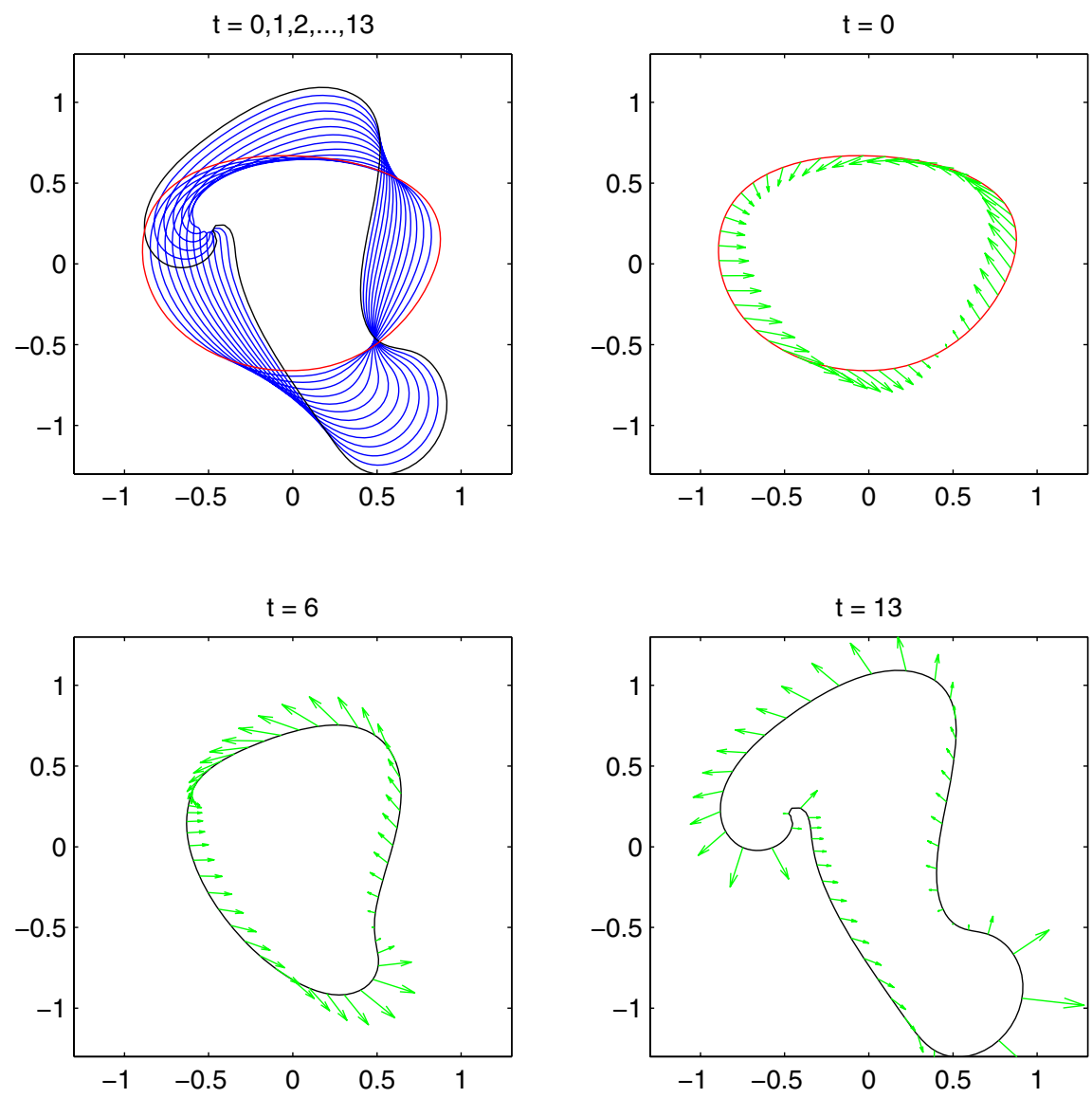

Figure 14. Motion of a momentum sheet with noncircular initial conditions, $H^{1}$ metric. The initial conditions are the same as in Figure 13.

Some dynamics of momentum sheets for initial conditions far from circular are shown in Figure 13 for the Gaussian kernel $G(r)=\exp \left(-(r / 0.2)^{2}\right)$. The momentum in the northwest corner of the sheet, initially pointing inward, swings around to point outward, forming an outwardly expanding bubble, a fairly stable object much like the circular sheets examined above. Another bubble is seen in the southeast corner. However, two other sections of the sheet do not succeed in turning around, and at $t=40$ are approaching each other (later they will zipper up as in Figure 8). The transition from outward to inward momentum at the southwest corner seems to be unstable and leads to the development of a growing finger.

The initial conditions in Figure 14 are the same as in Figure 13, but we now switch to the $H^{1}$ kernel $G(r)=K_{0}(r / 0.2)$. Apart from the time-scale, the motion is broadly similar 
until some fine structure emerges on the length-scale of the kernel. Shortly after the final time shown, the motion ends in a self-collision of the sheet.

Acknowledgment. We would like to thank the referees for their comments.

\section{REFERENCES}

[1] V. I. ARNOLD, Sur la géométrie différentielle des groupes de Lie de dimension infinie et ses applications à l'hydrodynamique des fluides parfaits, Ann. Inst. Fourier (Grenoble), 16 (1966), pp. 319-361.

[2] V. I. ARnold And B. A. Khesin, Topological Methods in Hydrodynamics, Appl. Math. Sci. 125, SpringerVerlag, New York, 1998.

[3] R. E. Caflisch, T. Y. Hou, AND J. Lowengrub, Almost optimal convergence of the point vortex method for vortex sheets using numerical filtering, Math. Comp., 68 (1999), pp. 1465-1496.

[4] E. Hairer, C. Lubich, And G. Wanner, Geometric Numerical Integration: Structure-Preserving Algorithms for Ordinary Differential Equations, Springer-Verlag, Berlin, 2002.

[5] D. D. Holm And J. E. Marsden, Momentum maps and measure-valued solutions (peakons, filaments and sheets) for the EPDiff equation, in The Breadth of Symplectic and Poisson Geometry, Progr. Math. 232, Birkhäuser Boston, Boston, MA, 2005, pp. 203-235.

[6] D. D. Holm, V. Putkaradze, and S. N. Stechman, Rotating concentric circular peakons, Nonlinearity, 17 (2004), pp. 1-24.

[7] D. D. Holm, J. T. Ratnanather, A. Trouvé, and L. Younes, Soliton dynamics in computational anatomy, NeuroImage, 23 (2004), pp. S170-S178.

[8] D. D. Holm and M. F. Staley, Wave structure and nonlinear balances in a family of evolutionary PDEs, SIAM J. Appl. Dyn. Syst., 2 (2003), pp. 323-380.

[9] D. D. Holm and M. F. Staley, Interaction Dynamics of Singular Wave Fronts, manuscript.

[10] T. Y. Hou, J. S. Lowengrub, And M. J. Shelley, The long-time motion of vortex sheets with surface tension, Phys. Fluids, 9 (1997), pp. 1933-1954.

[11] D. D. Holm, M. Nitsche, And V. Putkaradze, Euler-alpha and vortex blob regularization of vortex filament and vortex sheet motion, J. Fluid Mech., 555 (2006), pp. 149-176.

[12] B. Khesin, Topological fluid dynamics, Notices Amer. Math. Soc., 52 (2005), pp. 9-19.

[13] R. Krasny, A study of singularity formation in a vortex sheet by the point-vortex approximation, J. Fluid Mech., 167 (1986), pp. 65-93.

[14] A. J. Majda And A. L. BertozzI, Vorticity and Incompressible Flow, Cambridge University Press, Cambridge, UK, 2002.

[15] J. E. Marsden and T. S. Ratiu, Introduction to Mechanics and Symmetry: A Basic Exposition of Classical Mechanical Systems, 2nd ed., Springer-Verlag, Berlin, 1999.

[16] S. Marsland and C. Twining, Constructing diffeomorphic representations for the group-wise analysis of non-rigid registrations of medical images, IEEE Trans. Medical Imaging, 23 (2004), pp. 10061020.

[17] R. I. McLachlan and S. R. Marsland, $N$-particle dynamics of the Euler equations for planar diffeomorphisms, Dynam. Systems, to appear.

[18] R. I. McLachlan and G. R. W. Quispel, Geometric integrators for ODEs, J. Phys. A, 39 (2006), pp. $5251-5286$.

[19] M. I. Miller, A. Trouvé, And L. Younes, On metrics and the Euler-Lagrange equations of computational anatomy, Ann. Rev. Biomed. Engrg., 4 (2002), pp. 375-405.

[20] D. W. Moore, The spontaneous appearance of a singularity in the shape of an evolving vortex sheet, Proc. Roy. Soc. London Ser. A, 365 (1979), pp. 105-119.

[21] D. W. Moore And R. Griffiths-Jones, Stability of an expanding circular vortex sheet, Mathematika, 21 (1974), pp. 128-133.

[22] D. Mumford, Pattern theory: The mathematics of perception, in Proceedings of the International Congress of Mathematicians, Vol. III, World Scientific, Singapore, 2002, pp. 401-422.

[23] M. Nitsche, Axisymmetric vortex sheet motion: Accurate evaluation of the principal value integral, SIAM J. Sci. Comput., 21 (1999), pp. 1066-1084. 
[24] M. Oliver And S. ShKoller, The vortex blob method as a second-grade non-Newtonian fluid, Comm. Partial Differential Equations, 26 (2001), pp. 295-314.

[25] M. A. Pinsky, Introduction to Fourier Analysis and Wavelets, Brooks/Cole, Pacific Grove, CA, 2002.

[26] A. Sidi AND M. IsRAELI, Quadrature methods for periodic singular and weakly singular Fredholm integral equations, J. Sci. Comput., 3 (1988), pp. 201-231. 